\title{
Self-Similar Conformations and Dynamics in Entangled Melts and Solutions of Nonconcatenated Ring Polymers
}

\author{
Ting Ge ${ }^{\dagger}$, Sergey Panyukov ${ }^{\ddagger}$, and Michael Rubinstein ${ }^{*} \dagger$ \\ tDepartment of Chemistry, University of North Carolina, Chapel Hill, North Carolina 27599, \\ United States \\ ‡P. N. Lebedev Physics Institute, Russian Academy of Sciences, Moscow, Russia 117924
}

\section{Abstract}

A scaling model of self-similar conformations and dynamics of nonconcatenated entangled ring polymers is developed. Topological constraints force these ring polymers into compact conformations with fractal dimension $d_{\mathrm{f}}=3$ that we call fractal loopy globules (FLGs). This result is based on the conjecture that the overlap parameter of subsections of rings on all length scales is the same and equal to the Kavassalis-Noolandi number $O_{\mathrm{KN}} \approx 10-20$. The dynamics of entangled rings is self-similar and proceeds as loops of increasing sizes are rearranged progressively at their respective diffusion times. The topological constraints associated with smaller rearranged loops affect the dynamics of larger loops through increasing the effective friction coefficient but have no influence on the entanglement tubes confining larger loops. As a result, the tube diameter defined as the average spacing between relevant topological constraints increases with time $t$, leading to "tube dilation". Analysis of the primitive paths in molecular dynamics simulations suggests a complete tube dilation with the tube diameter on the order of the time-dependent characteristic loop size. A characteristic loop at time $t$ is defined as a ring section that has diffused a distance equal to its size during time $t$. We derive dynamic scaling exponents in terms of fractal dimensions of an entangled ring and the underlying primitive path and a parameter characterizing the extent of tube dilation. The results reproduce the predictions of different dynamic models of a single nonconcatenated entangled ring. We demonstrate that traditional generalization of single-ring models to multi-ring dynamics is not self-consistent and develop a FLG model with self-consistent multi-ring dynamics and complete tube dilation. This selfconsistent FLG model predicts that the longest relaxation time of nonconcatenated entangled ring polymers scales with their degree of polymerization $N$ as $\tau_{\text {relax }} \sim N^{7 / 3}$, while the diffusion coefficient of these rings scales as $D_{3 \mathrm{~d}} \sim$ $N^{-5 / 3}$. For the entangled solutions and melts of rings, we predict power law stress relaxation function $G(t) \sim t^{-3 / 7}$ at $t<\tau_{\text {relax }}$ without a rubbery plateau and the corresponding viscosity scaling

\footnotetext{
*Corresponding Author:mr@unc.edu (M.R.).

Supporting Information

The Supporting Information is available free of charge on the ACS Publications website at DOI: 10.1021/acs.macromol.5b02319. Analysis of stress relaxation functions in experiments and molecular dynamics simulations; comparison of the $s$-dependent and $t$ dependent primitive paths; calculations of the characteristic loop size; scaling description of the mean-square displacement of monomers and simulation results; discussion of the error bars of scaling exponents in simulations; details about measuring diffusion coefficients in simulations (PDF)

Notes

The authors declare no competing financial interest.
} 
with the degree of polymerization $N$ as $\eta \sim N^{4 / 3}$. These theoretical predictions are in good agreement with recent computer simulations and are consistent with experiments of melts of nonconcatenated entangled rings.

\section{Graphical abstract}
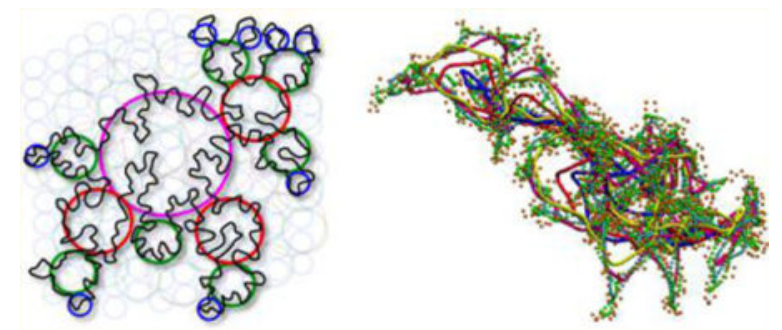

\section{INTRODUCTION}

One of the most important properties of polymers is that they are able to heavily overlap with each other ${ }^{1}$ and are characterized by large overlap parameter

$$
O=\frac{c R^{3}}{N} \gg 1
$$

defined as the number of molecules with $N$ Kuhn segments of length $b$ sharing the same pervaded volume $R^{3}$, where $c$ is the number of Kuhn segments per unit volume. The overlap parameter can be as high as $O \approx N^{1 / 2} b^{3} / v$ for linear chains of size $R \approx b N^{1 / 2}$ in the melt ( $c$ $\approx 1 / v$, where $V$ is the volume of a Kuhn monomer). ${ }^{1}$ Here and below we drop numerical coefficients on the order of unity and keep the discussion in the paper at the scaling level.

\subsection{Unentangled Polymer Dynamics}

Linear polymers do not topologically restrict motion of their neighbors if the overlap parameter is below the magic Kavassalis-Noolandi number ${ }^{1-4}$

$$
O_{\mathrm{KN}} \approx \frac{N_{e}^{1 / 2} b^{3}}{v} \approx 10-20
$$

where $N_{\mathrm{e}}$ is the number of Kuhn segments per entanglement strand. The polymer dynamics in this case $\left(O<O_{\mathrm{KN}}\right)$ is described by the Rouse model for melts and Rouse-Zimm model for semidilute unentangled solutions. ${ }^{1,5}$ A polymer chain is represented in these models by $N$ beads connected by springs with root-mean-square size $b$. The friction coefficient $\zeta$ of each bead in the Rouse model is assumed to be independent of the motion of other beads (no hydrodynamic coupling), and the friction coefficient of the entire chain consisting of $N$ beads is $\zeta_{\mathrm{R}} \approx N \zeta$. The diffusion coefficient of the unentangled polymer in a melt is obtained using the Einstein relation 6

$$
D_{\mathrm{R}} \approx \frac{k T}{N \zeta}
$$


and is reciprocally proportional to the number of beads-degree of polymerization $N$ independently of the chain architecture (linear, branched, ring, etc.), where $k T$ is the thermal energy ( $k$ is the Boltzmann constant and $T$ is the absolute temperature). The Rouse relaxation time is on the order of the time it takes a polymer to diffuse its size $R$

$$
\tau_{\mathrm{R}} \approx \frac{R^{2}}{D_{\mathrm{R}}} \approx \frac{\zeta N R^{2}}{k T}
$$

If the equilibrium conformation of a polymer chain (which could in principle have a complex branched architecture, e.g., randomly branched polymer near gel point ${ }^{1}$ ) is a fractal with fractal dimension $d_{\mathrm{f}}$, the chain size is $R \approx b N^{1 / d_{\mathrm{f}}}$ and the Rouse relaxation time of an unentangled polymer in a melt is

$$
\tau_{\mathrm{R}} \approx \tau_{0} N^{1+2 / d_{\mathrm{f}}} \sim N R^{2}
$$

where the monomeric relaxation time is $\tau_{0} \approx \zeta b^{2} /(k T)$. The fractal dimension of linear chains in a melt is $d_{\mathrm{f}}=2$, and their Rouse time is $\tau \approx \tau_{0} N^{2}$. We stress that Rouse model predicts self-similar (fractal) chain dynamics: each section of a chain consisting of $g$ monomers with section size $r \approx b g^{1 / d_{\mathrm{f}}}$ relaxes during time

$$
\tau(g) \approx \tau_{0} g^{1+2 / d_{\mathrm{f}}} \sim g r^{2}
$$

\subsection{Entangled Polymer Dynamics}

Polymers with higher overlap parameter $O>O_{\mathrm{KN}}$ strongly affect motion of their neighbors and are called entangled. ${ }^{1}$ In the classical tube model, proposed by Edwards ${ }^{7}$ for polymer networks with permanent topological interactions, each linear network strand is permanently confined by its neighbors to a tube-like region. ${ }^{8}$ de Gennes extended this idea of Edwards to dynamics of a free linear chain in an array of fixed topological obstacles, ${ }^{9}$ such as linear chains diffusing through a permanent network. de Gennes suggested that the motion of such polymers is also restricted to confining tubes, although topological constraints in this case are not permanent and the new tube is formed as the polymer diffuses out of the old one. ${ }^{1,5,8}$ He proposed reptation model in which polymer has hard time moving in directions normal to the axis of its tube and instead slides (reptates) predominantly along it. ${ }^{9}$ This motion along the contour of the tube is unhindered by the topological constraints and proceeds with Rouse curvilinear diffusion constant (eq 3) $D_{\mathrm{c}} \sim 1 / N$. The contour length $L$ of the Edwards tube, called primitive path, is proportional to chain length $N b$ of linear polymer $L \sim N$. Therefore, the time it takes for a linear chain to diffuse out of its original tube, called reptation time, is $1,8,9$

$$
\tau_{\text {rep }} \approx \frac{L^{2}}{D_{\mathrm{c}}} \sim N^{3}
$$

The reptation model predicts 3-dimentional diffusion coefficient ${ }^{1,5,8,9}$ 


$$
D_{3 \mathrm{~d}} \approx \frac{R^{2}}{\tau_{\text {rep }}} \sim N^{-2}
$$

Doi and Edwards ${ }^{10-13}$ generalized the de Gennes reptation model from a single chain in an array of fixed obstacles to melts of linear polymers. They predicted ${ }^{1,5,8,10}$ the viscosity $\eta$ of entangled liquids (such as melts) of linear chains to be proportional to the cube of polymer molecular weight $M$

$$
\eta \sim \tau_{\text {rep }} \sim M^{3}
$$

and their self-diffusion coefficient to have similar degree of polymerization dependence to that of a linear chain in an array of fixed obstacles (eq 8). Experimentally measured exponents for molecular weight dependence of viscosity and relaxation time were slightly larger than predicted by the reptation model (eq 9). ${ }^{14}$ The difference between experimentally measured exponent 3.4 and theoretically predicted exponent 3 is believed to be primarily due to tube length fluctuations introduced by Doi. ${ }^{15,16}$ Similar corrections to the reptation prediction were observed for diffusion coefficient ${ }^{17} D_{3 \mathrm{~d}} \sim M^{-2.3}$. Extensions of these models account for many-chain effects, such as constraint release ${ }^{18,19}$ and tube dilation, ${ }^{20}$ that are especially important in polydisperse systems ${ }^{21}$ and for branched entangled polymers. ${ }^{22}$

Tube dilation refers to the increase of the effective tube diameter during the relaxation of entangled polymer chains. This concept was introduced by Marrucci ${ }^{20}$ based on the consideration that the topological constraints giving rise to the tube can be effectively released due to the mobility of surrounding polymer chains. Marrucci described the chain segments relaxed from the tube as effective solvent that dilutes the topological constraints relevant to long-time chain dynamics and hence increases the effective tube diameter. The stress relaxation modulus in this model was predicted ${ }^{20,21}$ to scale as the square of the fraction $\varphi(t)$ of the polymer chain that remains in the effective tube at time $t$

$$
G(t) \approx G_{\mathrm{e}}[\phi(t)]^{2}, \text { for } t>\tau_{\mathrm{e}}
$$

where $G_{\mathrm{e}} \sim 1 / N_{\mathrm{e}}$ is the entanglement plateau modulus and $\tau_{\mathrm{e}} \sim N_{\mathrm{e}}^{2}$ is the relaxation time of an entanglement strand containing $N_{\mathrm{e}}$ monomers. The stress relaxation in eq 10 is faster than that in the original reptation model ${ }^{5}$ with $G(t) \approx G_{\mathrm{e}} \varphi(t)$. This approach to tube dilation called "double reptation" successfully characterized the stress relaxation in both polydisperse linear polymers ${ }^{20}$ and branched polymers, ${ }^{22}$ with narrow tube diameter at short times and wide tube diameter at long times. An alternative approach to describe tube dilation uses dilation exponent ${ }^{23,24} 7 / 3$ instead of 2 in eq 10. However, these approaches are not selfconsistent because they assume that tube dilation does not affect single chain motion in the "skinny" bare tube even at long times and does not renormalize the monomeric friction coefficient.

We emphasize that reptation dynamics is not self-similar because new conformations of the tube appear only at two chain ends. This leads to nonfractal (almost exponential) stress relaxation dominated by chain ends. Therefore, complete relaxation of a typical chain 
section containing $g$ monomers occurs on the same time scale as of the whole chain. The only exceptions are tube length fluctuations proposed by Doi ${ }^{15,16}$ that relax small sections near chain ends, each corresponding to a fraction $\left(N_{\mathrm{e}} / N\right)^{1 / 2}$ of the whole chain, by Rouselike self-similar motion along the tube. ${ }^{1}$

\subsection{Overview of Nonconcatenated Entangled Ring Polymers}

The success of the reptation model of linear entangled polymers ${ }^{22}$ emphasizes our failure to understand the dynamics of nonconcatenated entangled ring polymers that do not have ends ${ }^{25}$ and thus do not reptate like linear chains. Below we show that both conformations and dynamics of these ring polymers are self-similar because new conformations can appear on all length scales anywhere along ring contour. ${ }^{26}$ Besides obvious intellectual importance, strands with conformations and dynamics similar to those in nonconcatenated rings are also expected to occur in deswollen gels ${ }^{27}$ and thus influence properties of the gel networks.

Strong similarity has been suggested between conformations of nonconcatenated entangled rings in melts and packing of chromatin in nucleus ${ }^{28}$ with topological constraints forcing DNA molecules into fractal crumpled globule conformation. ${ }^{29}$ The original idea of fractal crumpled globule formed by collapsed linear chain was proposed by de Gennes ${ }^{30}$ and later by Grosberg, Nechaev, and Shakhnovich. ${ }^{31}$ They suggested that if the solvent condition suddenly deteriorates, a polymer chain collapses in a self-similar way, forming a kinetically trapped fractal globule. The structure of this crumpled globule depends on the kinetics of collapse and its relaxation toward equilibrium is slowed down by self-entanglements. The equilibrated globule of a linear chain is nonfractal. ${ }^{32}$

We would like to emphasize the difference between temporary topological constraints of linear chains (as well as tree-like branched polymers without loops) in entangled polymer solutions and melts ${ }^{33}$ (Figure 1a) and permanent topological constraints between ring polymers (Figure 1c,d). The equilibrium polymer conformations of the former are independent of these temporary topological constraints. Equilibrium conformations of both unentangled $\left(N<N_{\mathrm{e}}\right)$ and entangled $\left(N>N_{\mathrm{e}}\right)$ linear chains in melts and thetasolutions are almost ideal. ${ }^{1,8,32}$ The deviations of linear chain conformations from ideality are not related to the topological constraints. ${ }^{34}$ In contrast to linear polymers, the equilibrium conformations of ring polymers strongly depend on the topological constraints they impose on each other. Unentangled ring polymers with $N<N_{\mathrm{e}}$ are almost ideal in melts and theta solutions similar to their linear counterparts. Conformations of longer rings with $N>N_{\mathrm{e}}$ are strongly perturbed by topological constraints.

Equilibrium entanglement network formed by temporary entanglements between linear chains in solutions depends on chain flexibility, polymer concentration, and solvent quality but is independent of the preparation conditions (does not depend on the history of the sample). For ring polymers, there are permanent topological constraints such as self-knots (Figure 1b) and concatenation (Figure 1c), which are fixed at the time of synthesis and conserved as long as main chain bonds of polymers are not broken. 


\subsubsection{Double-Folded Lattice Animal (DFLA) Model}

Conformations: The lattice animal model of equilibrium conformation of a

nonconcatenated ring in an array of fixed topological obstacles was developed in mid-1980s..$^{35,36}$ In order to maximize the entropy of the ring, while preserving its topological constraints with a fixed lattice of obstacles, the ring preservation of topologyabsence of concatenation by obstacles - while randomly branched structure of double-folds (called lattice animals or, more precisely, lattice trees) maximizes entropy of these conformations. Self-interactions of different overlapping subsections of double-folded lattice animal (DFLA) conformations were ignored in this model of nonconcatenated rings in an array of fixed obstacles. The predicted fractal dimension of ideal lattice animals $d_{\mathrm{f}}=4$ is higher than space dimension $d=3$. Very large DFLA conformations of rings would become too dense and do not fit in three-dimensional space. Therefore, one would expect a crossover from $d_{\mathrm{f}}=4$ to space dimension $d=3$ as ring concentration approaches the bulk value $c \approx$ $1 / v$ for the number of Kuhn segments in a ring $N \approx N_{\mathrm{e}}^{3} b^{12} / v^{4}$, where $N_{\mathrm{e}}$ is the number of Kuhn segments in an entanglement strand with size on the order of the distance between topological obstacles $a \approx b N_{\mathrm{e}}^{1 / 2}$ and $v$ is the volume of a Kuhn monomer.

It was not clear how to extend the DFLA model to describe the conformations of many nonconcatenated rings in entangled solutions or melts. The DFLA model certainly describes the minimally open (maximally compact) conformation with no topological concatenations formed by double-folded sections of neighboring DFLA rings (Figure 1d). It was clear that the entropy of the system can be increased by opening a fraction of small loops of some rings and letting the unopened smallest loops of other rings penetrate through them. ${ }^{27} \mathrm{~A}$ similar operation can be performed on larger length scales, but how much penetration can be allowed on these length scales was not clear. If such penetration of loops into loops proceeds too far, the possibility of topological glass transition was suggested. ${ }^{27}$

Dynamics: Self-similar amoeba-like dynamics of non-concatenated rings in an array of fixed obstacles (DFLA model) was proposed ${ }^{35}$ in 1986 and corrected and tested by Monte Carlo simulations ${ }^{37}$ in 1994. The main idea of this fractal dynamics is that smaller loops diffuse along larger loops in a self-similar way. For any ring section of $g$ monomers (e.g., green line in Figure 2a) one can define the corresponding backbone, called primitive path (red line $\mathrm{AB}$ in Figure 2), by reducing the length of this section to the shortest topologically equivalent line through the array of obstacles. As a result, this ring section consists of the primitive path $\mathrm{AB}$ and a set of double-folded loops connected to it. This section (green line in Figure 2a) can be described as a fractal consisting of $g / N_{\mathrm{e}}$ elements of size $a \approx b N_{\mathrm{e}}^{1 / 2}$ and the section size

$$
r \approx a\left(\frac{g}{N_{\mathrm{e}}}\right)^{1 / d_{\mathrm{f}}}
$$

where the fractal dimension $d_{\mathrm{f}}=4$. The same distance $r$ between points $\mathrm{A}$ and $\mathrm{B}$ can also be expressed as the end-to-end distance of the primitive path (red line AB in Figure 2) with the fractal dimension $d_{\mathrm{p}}=2$ 


$$
r \approx a\left(\frac{l}{a}\right)^{1 / d_{\mathrm{p}}}
$$

corresponding to an ideal random walk with step size $a$ along the primitive path with contour length 1 . From eqs 11 and 12, the contour length of the primitive path is

$$
l \approx a\left(\frac{g}{N_{\mathrm{e}}}\right)^{d_{\mathrm{p}} / d_{\mathrm{f}}}
$$

with the exponent $d_{\mathrm{p}} / d_{\mathrm{f}}=1 / 2$. For an entangled linear chain with $d_{\mathrm{f}}=2$ and $d_{\mathrm{p}}=2$, the primitive path length $l \approx a\left(g / N_{\mathrm{e}}\right) \approx b g / N_{\mathrm{e}}{ }^{1 / 2}$ is proportional to the length $s \approx b g$ of the chain section of $g$ monomers. In contrast, the primitive path length for a section of $g$ monomers along a ring in the DFLA model with $d_{\mathrm{f}}=4$ and $d_{\mathrm{p}}=2$ is

$$
l \approx a\left(\frac{g}{N_{\mathrm{e}}}\right)^{1 / 2} \approx b g^{1 / 2} \ll \frac{b g}{N_{\mathrm{e}}^{1 / 2}}
$$

indicating that most of the mass of the ring section is in the double-folded loops. Therefore, a small fraction

$$
\frac{l / a}{g / N_{\mathrm{e}}} \approx\left(\frac{N_{\mathrm{e}}}{g}\right)^{1 / 2}
$$

of entanglement strands with $N_{\mathrm{e}}$ monomers in each (cyan loops in Figure 2b) move along the primitive path of the ring section consisting of $g$ monomers (red line in Figure 2b), resulting in the effective mass transport on this length scale through the entanglement network of fixed obstacles, whereas most of the entanglement strands (magenta loops in Figure $2 b$ ) move along the double-folded sections leading to ineffective displacement of mass on this length scale (it could still be effective on smaller length scales). The same partition of effective vs ineffective motion occurs on every length scale and results in the self-similar dynamics in the DFLA model. ${ }^{37}$

During the relaxation time of an entanglement strand

$$
\tau_{\mathrm{e}} \approx \tau_{0} N_{\mathrm{e}}^{2}
$$

the strand moves by its size $a \approx b N_{\mathrm{e}}^{1 / 2}$. This displacement of a single entanglement strand causes mean-square curvilinear displacement of the center of mass of a section with $g$ Kuhn monomers (green line in Figure 2a) by distance $\left(a \mathrm{~N}_{\mathrm{e}} / g\right)^{2}$. The curvilinear displacement of the center of mass of this section along its primitive path during time $\tau_{\mathrm{e}}$ produced by the diffusion of $1 / a$ minimal loops along the same primitive path is $\left(a N_{\mathrm{e}} / g\right)^{2}(I / a)$. The resulting curvilinear diffusion coefficient of this section of $g$ Kuhn monomers along its primitive path of length $l \approx a\left(g / N_{\mathrm{e}}\right)^{1 / 2}$ is

$$
D_{\mathrm{c}}(g) \approx D_{\mathrm{e}}\left(\frac{N_{\mathrm{e}}}{g}\right)^{2} \frac{l}{a} \approx D_{\mathrm{e}}\left(\frac{N_{\mathrm{e}}}{g}\right)^{3 / 2}
$$


where the Rouse diffusion coefficient $D_{\mathrm{e}}$ of a minimal entanglement loop with $N_{\mathrm{e}}$ Kuhn monomers (see eq 3 ) is

$$
D_{\mathrm{e}} \approx \frac{a^{2}}{\tau_{\mathrm{e}}} \approx \frac{k T}{N_{\mathrm{e}} \zeta}
$$

The time it takes the section of $g$ Kuhn monomers to diffuse its size is on the order of its relaxation time

$$
\tau(g) \approx \frac{l^{2}}{D_{\mathrm{c}}(g)} \approx \tau_{\mathrm{e}}\left(\frac{g}{N_{\mathrm{e}}}\right)^{5 / 2}
$$

Thus, the DFLA model predicts that the relaxation time of a nonconcatenated ring with $N$ Kuhn monomers is

$$
\tau_{\text {relax }} \approx \tau_{\mathrm{e}}\left(\frac{N}{N_{\mathrm{e}}}\right)^{5 / 2}
$$

Stress Relaxation: Kapnistos et al. ${ }^{38}$ derived the stress relaxation modulus for a single nonconcatenated ring in the DFLA model. The stress is supported by the gate of entanglements through which loops have not yet been pulled out, and thus the stress relaxation modulus is on the order of $k T$ per such gate of entanglements. (Such a gate is between two neighboring fixed obstacles.) The number density of gates associated with the loops containing $g \approx m N_{\mathrm{e}}$ monomers each is proportional to $m^{-3 / 2}$ for $1 \ll m \ll N / N_{\mathrm{e}}$. ${ }^{35-37}$ The lifetime of a loop of $g \approx m N_{\mathrm{e}}$ monomers and hence the relaxation time of the corresponding gate is $\tau(m) \approx \tau_{\mathrm{e}} m^{\beta}$ with $\beta=5 / 2$ (see eq 19). As a result, the single-ring stress relaxation modulus in the DFLA model is

$$
\begin{aligned}
G_{\mathrm{S}}(t) & \approx G_{\mathrm{e}} \phi(t) \approx G_{\mathrm{e}} \int m^{-3 / 2} \exp \left[-\frac{t}{\tau(m)}\right] \mathrm{d} m \\
& \approx G_{\mathrm{e}}\left(\frac{t}{\tau_{\mathrm{e}}}\right)^{-1 /(2 \beta)} \approx G_{\mathrm{e}}\left(\frac{t}{\tau_{\mathrm{e}}}\right)^{-1 / 5}
\end{aligned}
$$

where $\varphi(t) \approx\left(t / \tau_{\mathrm{e}}\right)^{-1 /(2 \beta)}$ is the fraction of unrelaxed gates at time $t$ in the interval $\tau_{\mathrm{e}}<t<$ $\tau_{\text {relax }}$. Milner and Newhall ${ }^{39}$ calculated the stress relaxation modulus $G_{\mathrm{s}}(t)$ in the DFLA model using essentially the same method. They estimated from their Monte Carlo simulations that the exponent $\beta \approx 8 / 3$ for the scaling relation between the relaxation time of a gate and the number $m$ of the associated entanglement strands and obtained the single-ring stress relaxation modulus $G_{\mathrm{s}}(t) \approx G_{\mathrm{e}}\left(t / \tau_{\mathrm{e}}\right)^{-1 /(2 \beta)} \approx G_{\mathrm{e}}\left(t / \tau_{\mathrm{e}}\right)^{-3 / 16}$ with the exponent $3 / 16 \approx$ 0.19 close to the value $1 / 5=0.2$ (eq 21) derived by Kapnistos et al. ${ }^{38}$

The single-ring stress relaxation modulus $G_{\mathrm{s}}(t)$ derived by Kapnistos et al. ${ }^{38}$ is shown schematically in Figure 3 (red line). At $t \approx \tau_{\text {relax }} \approx \tau_{\mathrm{e}}\left(N / N_{\mathrm{e}}\right)^{5 / 2}$

$$
G_{\mathrm{s}} \approx G_{\mathrm{e}}\left(\frac{N_{\mathrm{e}}}{N}\right)^{1 / 2} \approx \frac{\left(N / N_{\mathrm{e}}\right)^{1 / 2} k T}{v N}
$$


corresponding to $\left(N / N_{\mathrm{e}}\right)^{1 / 2}$ remaining stress-carrying entanglement strands along the primitive path of the ring. This implies that the backbone of the double-folded lattice tree is not yet completely relaxed by the relaxation time $\tau_{\text {relax }}$ (eq 20). The stress relaxes exponentially at $t>\tau_{\text {relax }}$ as shown in Figure 3, in a manner similar to the terminal relaxation of entangled linear polymers after the entanglement stress plateau.

Extension of DFLA Model to Entangled Melts of Non-concatenated Rings: In a melt of entangled nonconcatenated rings, topological constraints are not fixed as in the DFLA model. As the sections of entangled rings progressively relax, the topological constraints are reorganized as well. Kapnistos et al. ${ }^{38}$ applied the approach of double reptation ${ }^{20}$ previously used for entangled linear and branched polymers to account for the dynamic reorganization of topological constraints between entangled rings. They estimated the multi-ring stress relaxation modulus for a melt of nonconcatenated rings based on eq 21 as

$$
G(t) \approx G_{\mathrm{e}}[\phi(t)]^{2} \approx G_{\mathrm{e}}\left(\frac{t}{\tau_{\mathrm{e}}}\right)^{-2 / 5}, \text { for } \tau_{\mathrm{e}}<t<\tau_{\text {relax }}
$$

which is shown schematically by the blue line in Figure 3. The same result can be obtained based on the picture that the multi-ring stress relaxation modulus in the time interval $\tau_{\mathrm{e}}<t<$ $\tau_{\text {relax }}$ is on the order of $k T$ per smallest unrelaxed loop ${ }^{1,38}$

$$
G(t) \approx \frac{k T}{v g} \approx \frac{k T}{v N_{\mathrm{e}}}\left(\frac{t}{\tau_{\mathrm{e}}}\right)^{-2 / 5} \approx G_{\mathrm{e}}\left(\frac{t}{\tau_{\mathrm{e}}}\right)^{-2 / 5}, \text { for } \tau_{\mathrm{e}}<t<\tau_{\text {relax }}
$$

where eq 19 for the relaxation time of a section with $g$ monomers in the DFLA model was used. However, the derivation in eq 24 is not self-consistent, as tube dilation is not considered in the derivation of $\tau(g)$ in eq 19.

Milner and Newhall ${ }^{39}$ have also estimated the multi-ring stress relaxation modulus $G(t)$ from their result for the single-ring stress relaxation modulus $G_{\mathrm{s}}(t) \approx G_{\mathrm{e}}\left(t \tau_{\mathrm{e}}\right)^{-1 /(2 \beta)} \approx G_{\mathrm{e}}\left(t / \tau_{\mathrm{e}}\right)^{-3 / 16}$ with their estimate of the exponent $\beta \approx 8 / 3$ and a tube dilation exponent that characterizes the dynamic reorganization of topological constraints $\gamma=7 / 3 .^{23,24}$ They obtained $G(t) \sim$ $\left[G_{\mathrm{s}}(t) / G_{\mathrm{e}}\right]^{\gamma} \sim t^{-7 / 16}$. However, this result is problematic, as it predicts a stress relaxation faster than that allowed by the rearrangements of loops. The multi-ring stress relaxation modulus at $\tau_{\mathrm{e}}<t<\tau_{\text {relax }}$ is at least $\sim k T$ per smallest unrelaxed loop with $m(t)$ entanglement strands, and therefore the fastest stress relaxation for their value of $\beta=8 / 3$ corresponds to $G(t) \sim 1 / m(t) \sim t^{1 / \beta} \sim t^{-3 / 8}$.

\subsubsection{Cates and Deutsch's Conjecture on Conformations of Ring Polymers-}

Cates and Deutsch ${ }^{40}$ have proposed a mean-field approach to determine the conformations of non-concatenated rings. They assumed that the free energy cost of topological interactions of a ring is thermal energy $k T$ per neighboring ring it overlaps with. Thus, the total topological free energy per chain is proportional to the overlap parameter $k T O \approx$ $k T R^{3} /(N V)$ (see eq 1). Balancing this topological free energy cost with confinement free energy penalty $k T N b^{2} / r^{2}$, Cates and Deutsch predicted partially compacted conformations of nonconcatenated rings with the ring size $R \sim N^{2 / 5}$. It is not clear why it was assumed that 
free energy cost of topological interactions per nonconcatenated ring is linearly proportional to the number of other rings it overlaps with. We will show below that this free energy cost is much higher —on the order of $k T$ per temporary entanglement $\sim k T N / N_{\mathrm{e}}$. The conformations of nonconcatenated ring polymers have also been studied by Sakaue ${ }^{41}$ using the mean-field approach and by Brereton and Vilgis, ${ }^{42}$ who considered a melt with zero winding number between each pair of rings.

1.3.3. Grosberg's Model-Grosberg ${ }^{43}$ assumed that a nonconcatenated ring in an entangled melt has a double-folded branched structure similar to the ring conformation in the DFLA model but is a compact object with fractal dimension $d_{\mathrm{f}}=3$ at the length scales above the spacing a between entanglements. The underlying skeleton of such an entangled ring is conjectured to be a lattice tree randomly branched at the scale of entanglement spacing $a$. Grosberg wrote down a Flory-type free energy for an entangled ring as a function of the length $L$ of the primitive path, which is the backbone of the randomly branched tree

$$
F \approx \frac{R^{2}}{a L}+\frac{L^{2}}{N b^{2}}
$$

where the size of the ring consisting of $N$ Kuhn monomers is

$R \approx a\left(N / N_{\mathrm{e}}\right)^{1 / d_{\mathrm{f}}} \approx a\left(N / N_{\mathrm{e}}\right)^{1 / 3}$. The first free energy term $r^{2} /(a L)$ arises as it is assumed that the ring is stretched to size $R$ larger than the ideal size of the randomly branched tree $a(L / a)^{1 / 2} \approx(a L)^{1 / 2}$, which corresponds to the ideal random-walk conformation of the primitive path. The term $L^{2} /\left(N b^{2}\right)$ was first derived by Gutin et al. ${ }^{44}$ as the entropic free energy of the random branching of a polymer with $N$ monomers and a contour length $L$ between two fixed ends. The same term is used in eq 25 to describe the random branching of the skeleton of an entangled ring. Note that the first term in eq 25 penalizes branching with smaller primitive path length $L$, whereas the second term promotes branching. The balance of the two terms yields $L \sim N^{5 / 9}$ and $R \sim L^{3 / 5}$. Grosberg argued that the scaling relation

$$
R \approx a(L / a)^{3 / 5}
$$

suggests that the random-walk conformation of the primitive path of a nonconcatenated ring follows the self-avoiding statistics with the fractal dimension $d_{\mathrm{p}}=5 / 3$ rather than the ideal Gaussian statistics with $d_{\mathrm{p}}=2$ as in the DFLA model (see eq 12).

The differences between Grosberg's model and the DFLA model are the values of the fractal dimension $d_{\mathrm{f}}$ of a nonconcatenated entangled ring and the fractal dimension $d_{\mathrm{p}}$ of the primitive path of a ring. Using Grosberg's values of $d_{\mathrm{f}}$ and $d_{\mathrm{p}}$, the single-ring dynamics in the DFLA model has been rederived and then employed ${ }^{45}$ to describe the stress relaxation in a melt of entangled rings. It was shown ${ }^{45}$ that in Grosberg's model the relaxation time of a section of $g$ Kuhn monomers $\tau_{\mathrm{g}} \sim g^{-2.56}$ and the multi-ring stress relaxation modulus is $G(t)$ $\approx G_{\mathrm{e}}\left(t / \tau_{\mathrm{e}}\right)^{-0.39}$, which is obtained based on the same argument ${ }^{1,38}$ that produced eq 24. However, this derivation of multi-ring stress relaxation modulus is also not self-consistent for the similar reason as the generalization of the DFLA model to melts of rings as it does not include the tube dilation in the derivation of $\tau_{\mathrm{g}}$ but assumes tube dilation for $G(t)$. 


\section{FRACTAL LOOPY GLOBULE (FLG) MODEL}

Existing scaling models for nonconcatenated entangled ring polymers provide different descriptions of the conformations of entangled rings and the corresponding primitive paths. The scaling of ring degree of polymerization $N$ with its size $R$ in Cates and Deutsch's theory is $N \sim R^{5 / 2}$ (section 1.3.2), while the fractal dimension of a ring in the DFLA model is $d_{\mathrm{f}}=4$ (section 1.3.1), and it is $d_{\mathrm{f}}=3$ in Grosberg's model (section 1.3.3). In section 2.1, we account for entanglements between rings on all length scales in a self-similar way ${ }^{26}$ and demonstrate that nonconcatenated entangled rings are fractal loopy globules (FLGs) with fractal dimension $d_{\mathrm{f}}=3$. The same fractal dimension has been obtained by Grosberg ${ }^{43}$ and Obukhov et al. ${ }^{46}$ as well. The primitive path of a ring section in the DFLA model (section 1.3.1) adopts the ideal random walk conformation with fractal dimension $d_{\mathrm{p}}=2$ between the two ends of this section while it was assumed by Grosberg (section 1.3.3) to be in a selfavoiding random walk conformation with fractal dimension $d_{\mathrm{p}}=5 / 3$. In section 2.2 , in light of the analysis of primitive paths in molecular dynamics simulations, we propose a scaling description of the primitive paths with complete tube dilation during the dynamics of entangled rings: the primitive path step size (tube diameter) is on the order of the timedependent characteristic loop size defined as the size of a ring section that has diffused a distance equal to its size. Compared with other models, our fractal loopy globule (FLG) model with fractal dimension $d_{\mathrm{f}}=3$ and complete tube dilation provides predictions in best agreement with simulation results. In this work, no independent new simulations of nonconcatenated ring melts have been performed. We reanalyze the results of the simulations by Halverson et al. ${ }^{47,48}$ to obtain our estimates of various scaling exponents in their simulations (see Table 1). However, we design and perform original primitive path analysis of one of the simulation samples provided by Halverson.

\subsection{Conformations of Nonconcatenated Entangled Rings}

The main concept of the self-similarity of entanglements on all length scales is that nonconcatenated rings and loops (sections of rings) can overlap with each other on different length scales without disturbing conformations of each other. On each length scale, the overlapping loops of corresponding size are characterized by constant Kavassalis-Noolandi overlap parameter $^{2-4} O_{\mathrm{KN}}$ (eq 2). Larger overlap of loops on a given length scale without significant distortion is prohibited by topological constraints. We emphasize that our description of the overlap of entangled loops on all length scales using $O_{\mathrm{KN}}$ is a conjecture and needs to be further tested by experiments and simulations.

To illustrate this concept, let us start from a dilute solution of large nonconcatenated rings in a theta solvent and increase their concentration by slowly evaporating solvent. The conformation of rings in a dilute theta-solvent solution is almost ideal with mean-square radius of gyration $\left\langle R_{\mathrm{g}}{ }^{2}\right\rangle=b^{2} N / 12$ equal to half of that of the corresponding linear chains. The overlap parameter of rings (eq 1) exceeds unity $O>1$ at polymer concentrations above the overlap concentration $c^{*} \sim N^{-1 / 2}$, but conformations of overlapping rings stay almost ideal and unperturbed by other rings as long as their concentration is below entanglement concentration $c_{\mathrm{e}}$ and the overlap parameter is below the Kavassalis-Noolandi value ${ }^{2-4}$ (for $c^{*}<c<c_{\mathrm{e}}$ and $\left.1<O<O_{\mathrm{KN}}\right)$. 
As solution concentration exceeds $c_{\mathrm{e}}$, rings can no longer stay ideal-like as condition of nonconcatenation would be violated. The conjecture we propose ${ }^{26}$ is that ring conformation changes into that of a large loop that stays at the overlap parameter $O_{\mathrm{KN}}$ with similar size loops of neighboring rings and a set of smaller loops at the same overlap parameter $O_{\mathrm{KN}}$ with similar size loops. The constant overlap $O_{\mathrm{KN}}$ of loops is conjectured to occur in a selfsimilar way over a wide range of length scales from an entanglement length $a(c)$ up to ring size $R$ (in a hyperscaling-like ${ }^{1,49}$ manner). The lower boundary of this fractal structure is on the order of tube diameter $a(c)$ of linear chains at concentration $c$ (Figure 4$)$. The upper boundary is the ring size

$$
R \approx\left(\frac{O_{\mathrm{KN}} N}{c}\right)^{1 / 3}
$$

which is $O_{\mathrm{KN}}{ }^{1 / 3}$ times larger than the distance $r_{\mathrm{cm}} \approx(c / N)^{-1 / 3}$ between centers of mass of neighboring rings to ensure that their overlap parameter (eq 1) stays at $O_{\mathrm{KN}}$ (eq 2). The fractal dimension of rings over these length scales $a(c)<r<R(c)$ is $d_{\mathrm{f}}=3$ (Figure $4 \mathrm{~b}$ ). This fractal loopy globule (FLG) conformation of nonconcatenated rings in entangled solutions with concentration $c>c_{\mathrm{e}}$ and melts (fat deer in Figure 4a) is somewhat analogous to DFLA conformation (skinny deer in Figure 2a)—randomly branched structure of loops on loops (cactus-like conformation)—but the distribution of branch sizes and probability of branching is different as clear from their different fractal dimensions. Another difference between the two models is that unlike the DFLA case loops in the loopy globule model are not perfect double folds.

Rosa and Everaers ${ }^{50}$ have examined the overlap parameter $O$ as a function of the ratio between the ring contour length $L_{\mathrm{r}} \sim N_{\mathrm{e}}$ and the entanglement length $L_{\mathrm{e}} \sim N_{\mathrm{e}}$ in their simulations of nonconcatenated rings in a melt. They found that the overlap parameter $O$ increases with the ratio $L_{\mathrm{T}} / L_{\mathrm{e}}$ and then remains close to the Kavassalis-Noolandi overlap parameter $O_{\mathrm{KN}} \approx 20$ at $L_{\mathrm{r}} / L_{\mathrm{e}}>10$. This finding provides direct evidence in support of the conjecture in the FLG model that entangled loops of different sizes overlap with similar size loops at the same overlap parameter $O_{\mathrm{KN}}$. Similar to the FLG model, Obukhov, Johner, Baschnagel, Meyer, and Wittmer ${ }^{46}$ have proposed a decorated loop model in which a nonconcatenated ring adopts the conformation of a tree-like structure made of a skeleton loop decorated by smaller loops of various sizes. Polydisperse loops in this model penetrate each other to fill the space while obeying the topological constraint of nonconcatenation as in the FLG model.

Below we restrict our consideration to nonconcatenated rings in melts $(c \approx 1 / v)$. Generalization of loopy globule model to solutions $\left(c_{\mathrm{e}}<c<1 / v\right)$ is straightforward. The size of any subsection of $g$ Kuhn segments in melts of linear polymers is Gaussian $r \approx b g^{1 / 2}$. Ring sections with $g<N_{\mathrm{e}}$ smaller than entanglement strands (entanglement scale $a \approx b N_{\mathrm{e}}{ }^{1 / 2}$ are still ideal-like (blue line with slope $1 / 2$ in Figure $4 \mathrm{~b}$ ). Larger subsections in loopy globule conformations are compressed with fractal dimension $d_{\mathrm{f}}=3$ and size 


$$
r \approx a\left(\frac{g}{N_{\mathrm{e}}}\right)^{1 / 3}, \text { for } a<r<R
$$

(red line with slope 1/3 in Figure 4b) up to the size $R$ of a loopy globule (eq 27) in a melt

$$
R \approx b N^{1 / 3} N_{\mathrm{e}}^{1 / 6}
$$

The number of loops of length scale $r$ per ring decreases reciprocally proportional to the pervaded volume $R^{3}$ of these loops

$$
n(r) \approx\left(\frac{R}{r}\right)^{3}
$$

The total number of loops per ring is proportional to the number of the smallest loops of size a

$$
\int_{r=a}^{r=R} \mathrm{~d}[n(r)] \approx R^{3} \int_{a}^{R} r^{-4} \mathrm{~d} r \approx n(a) \approx \frac{R^{3}}{a^{3}} \approx \frac{N}{N_{\mathrm{e}}}
$$

and is on the order of number of entanglement strands $N / N_{\mathrm{e}}$ per ring.

In their decorated loop model, Obukhov et al. ${ }^{46}$ constructed a mathematical formulation to delineate the decoration process. They calculated the mean-square radius of gyration $R_{\mathrm{g}}{ }^{2}$ of a ring as a function of the degree of polymerization $N$ and obtained $R_{\mathrm{g}}{ }^{2} \sim N^{2 / 3}(1-$ const $\left.\cdot N^{-1 / 3}\right)$. This result demonstrates that rings are asymptotically compact with $R_{\mathrm{g}}^{2} \sim N^{2 / 3}$ in the long chain limit $(N \rightarrow \infty)$ after a broad crossover regime extending to $N \sim 10^{3}-10^{4}$. The compactness of nonconcatenated entangled rings with fractal dimension $d_{\mathrm{f}}=3$ is also observed in recent molecular dynamics simulations. ${ }^{47,48}$

\subsection{Dynamics of Nonconcatenated Entangled Rings}

2.2.1. Primitive Path of a Nonconcatenated Entangled Ring-The description of the primitive path of a ring is at the core of a scaling theory for the dynamics of nonconcatenated entangled rings. During the entangled dynamics of rings, the primitive path of a ring section is revealed by reducing the ring section to the shortest segment that obeys all the dynamically relevant topological constraints imposed on the section. For the singlering calculation in the DFLA model ${ }^{35,37}$ and Grosberg's model, ${ }^{43}$ the topological constraints are permanent, and the primitive path of a ring section adopts an ideal ${ }^{35,37}$ or self-avoiding $^{43}$ random walk conformation with the step size equal to the fixed spacing a between topological constraints. For a melt of nonconcatenated entangled rings, the topological constraints contributing to the confining tubes are diluted as loops of increasing sizes are successively rearranged. Thus, the tube diameter, defined as the average spacing between topological constraints relevant to the suppression of fluctuations of ring sections at a particular time scale, increases with time during the dynamics of rings. If only part of the topological constraints associated with rearranged smaller loops are released, there is a partial tube dilation, in which the increase of the tube diameter is slower than the growth of the size of a characteristic loop that has diffused a distance equal to its size. In the limiting 
case, all the topological constraints related to rearranged smaller loops are released and result in a complete tube dilation; i.e., the tube diameter increases with time at the same rate as the characteristic loop size. With either partial or complete tube dilation, the conformation of the primitive path of a ring section is expected to be different from the random walk conformation in the DFLA model and Grosberg's model where topological constraints are permanent.

Analysis of Primitive Paths in Simulations: In order to reveal the primitive paths of ring polymers at different time and length scales in molecular dynamics simulations and thereby examine the extent of tube dilation during dynamics of entangled rings, we apply two primitive path determination methods. One method is developed based on the standard primitive path analysis (PPA) algorithm. ${ }^{51,52}$ We fix every sth monomer along the ring contour for each ring and then reduce the contour lengths of all rings simultaneously without allowing the crossing of ring sections as in the standard PPA algorithm. Since there are $s$ possible sets of fixed monomers, we determine the corresponding $s$ different reduced conformations for each ring. The average of the $s$ reduced conformations for a ring yields the primitive path of the ring with loops consisting of $s s$ monomers all pulled out. A primitive path obtained in this way is called an $s$-dependent primitive path ( $s$-PP). For one entangled ring in simulations, the original chain conformation and a set of $s$-PPs with different values of $s$ are visualized in Figure 4c, exhibiting features consistent with the FLG conformation as illustrated in Figure 4a.

Another method of revealing the primitive paths of entangled rings is through averaging the positions of monomers over a time interval $t^{53}$ During the dynamics of a melt of nonconcatenated rings, the conformation of a ring fluctuates around the primitive path while obeys all dynamically relevant topological constraints. Time averaging removes the fluctuations around the primitive path, and the line connecting the time-averaged positions of all monomers in a ring is interpreted as the primitive path. We call a primitive path obtained using this method a $t$-dependent primitive path $(t$-PP).

The results of the root-mean-square end-to-end distance $r$ of a primitive path segment as a function of the segment contour length $l$ are shown in Figures $5 \mathrm{a}$ and $5 \mathrm{~b}$ for a set of $s$-PPs and a set of $t$-PPs, respectively. Both sets of results are well fitted using the function

$$
r=2^{1 / \kappa} r_{\mathrm{c}}\left[\left(l / l_{\mathrm{c}}\right)^{-\kappa}+\left(l / l_{\mathrm{c}}\right)^{-\kappa \mu}\right]^{-1 / \kappa}
$$

which describes the crossover from $r \sim 1$ to $r \sim \mu^{\mu}$ with the crossover segment size $r_{\mathrm{c}}$ and crossover contour length $I_{\mathrm{c}}$ depending on $s$ or $t$, respectively. As shown in the insets of Figures $5 \mathrm{a}$ and $5 \mathrm{~b}$, the results of $r$ vs $I$ for the two sets of primitive paths can be collapsed onto their respective master curves using eq 32. We obtain $\kappa \approx 1.64 \pm 0.05$ and $\mu \approx 0.35$ \pm 0.01 for the $s$-PPs while $\kappa \approx 1.51 \pm 0.04$ and $\mu \approx 0.33 \pm 0.01$ for the $t$-PPs. Since the values of $\kappa$ and $\mu$ are close to each other for the two sets of primitive paths, the results of $t / r_{\mathrm{C}}$ vs $I / I_{\mathrm{c}}$ for all different primitive paths can be superimposed onto each other, as shown in Figure 5c. This result demonstrates that the conformations of the two sets of primitive paths both follow the scaling behavior $r \sim I$ for straight segments on length scales smaller than the 
time-dependent primitive path step (tube diameter) and $r \sim 1^{1 / 3}$ for compact objects on length scales larger than the time-dependent tube diameter and exhibit an almost identical crossover between the two scaling regimes for both methods of primitive path constructions.

We estimate the tube diameter as the crossover size $r_{\mathrm{c}}$ of a segment along the primitive path. The extent of tube dilation is determined by comparing the time dependences of the crossover segment size $r_{\mathrm{C}}$ and the root-mean-square end-to-end distance of a characteristic loop. The characteristic loop is defined as the ring section containing $g$ monomers such that its root-mean-square end-to-end distance $\left\langle r_{\mathrm{e}}^{2}(g)\right\rangle^{1 / 2}$ is equal to the root-mean-square displacement of its center of mass $\left\langle\Delta r_{\mathrm{CM}^{2}}(g, t)\right\rangle^{1 / 2}$ at time $t$

$$
r(g, t) \equiv\left\langle r_{\mathrm{e}}^{2}(g)\right\rangle^{1 / 2}=\left\langle\Delta r_{\mathrm{CM}}^{2}(g, t)\right\rangle^{1 / 2}
$$

which corresponds to a ring section that has diffused by its own size during time $t$. In section $\mathrm{C}$ of the Supporting Information, we present other measures of the characteristic loop size and show that the results of different measures agree with each other over many decades of time with $20 \%$ accuracy.

Time dependences of the tube diameter and the characteristic loop size measured respectively as $r_{\mathrm{c}}$ (see eq 32) and $r(g, t)$ (see eq 33) are shown in Figure 5d. At $t$ smaller than about $4000 \tau, r(g, t)<r_{\mathrm{c}}$, corresponding to the rearrangement of ring sections with sizes smaller than the entanglement size $a$ and hence a ring polymer is not yet "aware" of its confining tube. The intersection of $r_{\mathrm{c}}$ and $r(g, t)$ at $t \approx 4000 \tau$ suggests that the entanglement time $\tau_{\mathrm{e}}$ is about $4000 \tau$, at which entanglement strands have diffused by a distance of the undiluted tube diameter $a\left(\tau_{\mathrm{e}}\right) \approx a_{0}$. As $t$ exceeds $4000 \tau$, the tube diameter $a(t) \approx r_{\mathrm{c}}(t)$ increases with $t$ and follows the trend of the increase of the characteristic loop size $r(g, t)$ with $t$. This observation that the dilated tube diameter at $t>\tau_{\mathrm{e}}$ is on the order of the characteristic loop size indicates a complete tube dilation.

Our further analysis shows a one-to-one mapping between the two primitive path constructions, $s$-PP and $t$-PP. The root-mean-square difference $\delta$ between the position of a monomer along an $s$-PP and its corresponding position along the $t$-PP with the same crossover segment size $r_{\mathrm{c}}$ is calculated (see section B in the Supporting Information for the calculation details). The difference $\delta$ is proportional to but smaller than the corresponding $r_{\mathrm{C}}$ $\left(\delta / r_{\mathrm{c}} \approx 0.6-0.8\right.$ as shown in the inset of Figure $\left.5 \mathrm{~d}\right)$, indicating that the difference between the two primitive path constructions using different protocols is smaller than the tube diameter. Note that the primitive path is defined with the accuracy of tube diameter $a(t)$, and this difference between its two constructions with $\delta(t)<a(t)$ is not significant. The mapping between a $s$-PP and a $t$-PP reflects the equivalence between pulling taut any loops containing less than $s$ monomers and averaging monomer positions over a time interval $t$. The equivalence of the two primitive path construction methods is consistent with the picture that the primitive paths evolve and coarsen with time as loops of increasing size are progressively rearranged.

Scaling Description of Primitive Paths: Our analysis of the primitive paths in simulations shows that there is a complete tube dilation during the dynamics of entangled rings. The tube 
diameter increases with time such that it is on the order of the size $r(g, t)$ of a characteristic loop containing $g(t)$ monomers

$$
a(t) \approx r(g, t) \approx a_{0}\left[\frac{g(t)}{N_{\mathrm{e}, 0}}\right]^{1 / 3}, \tau_{\mathrm{e}}<t<\tau_{\text {relax }}
$$

with the undiluted tube diameter $a\left(\tau_{e}\right) \approx a_{0} \approx b\left(N_{e, 0}\right)^{1 / 2}$. Since the tube diameter $a(t)$ and the number of monomers $N_{e}(t)$ per tube segment are time dependent in the case of tube dilation, we denote their undiluted bare values respectively by $a_{0}$ and $N_{e, 0}$ rather than $a$ and $N_{e}$ as in the DFLA model and Grosberg's model with no tube dilation. The analysis of the primitive paths in simulations also reveals the conformation of primitive path (see Figure 5) in accordance with complete tube dilation. The primitive path of a characteristic loop is a single step (straight segment) of the confining tube with its size equal to the tube diameter $a(t)$, while the primitive path of a larger loop with $s$ monomers in an array of rearranged smaller loops of size $g(t)$ is a fractal with size

$$
r(s, t) \approx a(t)\left[\frac{l(s, t)}{a(t)}\right]^{1 / 3}, \text { for } g(t)<s<N \text { and } \tau_{\mathrm{e}}<t<\tau_{\text {relax }}
$$

where the fractal dimension is the same as that of a ring in the FLG conformation (see section 2.1), and $l(s, t)$ is the primitive path length at time $t$ of a section containing $s$ monomers. If the tube dilation were not complete such that the size of a characteristic loop is multiple times the tube diameter, the primitive path of this loop would have a different fractal structure on length scales between $a(t)$ and $r(g, t)$ (eq 34). This fractal dimension could be $d_{\mathrm{p}}=2$ by analogy with the DFLA model or $d_{\mathrm{p}}=5 / 3$ as suggested by Grosberg. ${ }^{43}$ We do not observe such regime in the primitive paths of rings in the molecular dynamics simulation data (see Figure 5). The fractal loopy globule (FLG) model with complete tube dilation (see eq 34) allows self-consistent calculations of the stress relaxation function and the melt viscosity of ring polymers as demonstrated in subsequent section 2.2.3. Therefore, we call the FLG model with complete tube dilation self-consistent FLG model, in contrast to the multi-ring DFLA model, Grosberg's model, and the "naive" FLG model, where complete tube dilation is introduced not self-consistently.

2.2.2. Relaxation Time and Diffusion Coefficient-We derive the relaxation time of a ring polymer with a quantitative description of tube dilation in the Appendix. The derivation reflects the self-similar nature of nonconcatenated ring dynamics and incorporates an appropriate renormalization of monomeric friction at different time scales, the lack of which has been a major criticism of the tube dilation models for star polymers, ${ }^{54}$ bidisperse blends of linear polymers, ${ }^{55}$ and star/linear polymer blends. ${ }^{56}$ We obtain a general result of the relaxation time of a ring containing $N$ monomers

$$
\tau_{\text {relax }} \approx \tau_{\mathrm{e}}\left(\frac{N}{N_{\mathrm{e}, 0}}\right)^{2+(1-\theta) d_{\mathrm{p}} / d_{\mathrm{f}}+\theta / d_{\mathrm{f}}}
$$

with $0 \leq \theta \leq 1$ (see eq A.19). The parameter $\theta$ quantifies the extent of tube dilation. During the entangled dynamics of rings, the number of monomers per entanglement strand $N_{\mathrm{e}}(t)$ 
scales with the number of monomers in a characteristic loop $g(t)$ as $N_{\mathrm{e}}(t) \sim[g(t)]^{\theta}$. In the limit $\theta=1$, there is complete tube dilation, and $N_{\mathrm{e}}(t) \approx g(t)$ meaning that an entanglement strand and a characteristic loop have comparable sizes. In the opposite limit $\theta=0$, there is no tube dilation, and $N_{\mathrm{e}}(t)$ is time independent and equal to $N_{\mathrm{e}, 0}$ for an entanglement strand at $t$ $\approx \tau_{\mathrm{e}}$. Based on eq 36, a general result of the 3-dimensional diffusion coefficient of nonconcatenated rings can be derived

$$
D_{3 \mathrm{~d}} \approx \frac{R^{2}}{\tau_{\mathrm{relax}}} \approx D_{\mathrm{e}}\left(\frac{N}{N_{\mathrm{e}, 0}}\right)^{\left.-2+\left[2-(1-\theta) d_{\mathrm{p}}-\theta\right)\right] / d_{\mathrm{f}}}
$$

where $D_{\mathrm{e}} \approx a_{0}^{2} / \tau_{\mathrm{e}}$ is the Rouse diffusion coefficient of an entanglement strand containing $N_{\mathrm{e}, 0}$ monomers (see eq 18).

If the tube dilation is complete with $\theta=1$, the relaxation time

$$
\tau_{\text {relax }} \approx \tau_{\mathrm{e}}\left(\frac{N}{N_{\mathrm{e}, 0}}\right)^{2+1 / d_{\mathrm{f}}}
$$

and the 3-dimensional diffusion coefficient

$$
D_{3 \mathrm{~d}} \approx D_{\mathrm{e}}\left(\frac{N}{N_{\mathrm{e}, 0}}\right)^{-2+1 / d_{\mathrm{f}}}
$$

are independent of the fractal dimension $d_{\mathrm{p}}$ of the primitive path. These results are consistent with the picture that a characteristic loop has a size on the order of the tube diameter and hence does not feel the fractal structure of the primitive path at length scales above the tube diameter. With $\theta=1$ and $d_{\mathrm{f}}=3$, the self-consistent FLG model predicts

$$
\tau_{\text {relax }} \approx \tau_{\mathrm{e}}\left(\frac{N}{N_{\mathrm{e}, 0}}\right)^{7 / 3}
$$

and

$$
D_{3 \mathrm{~d}} \sim N^{-5 / 3}
$$

as listed in Table 1 . If there is no tube dilation with $\theta=0$, one obtains.

$$
\tau_{\text {relax }} \approx \tau_{\mathrm{e}}\left(\frac{N}{N_{\mathrm{e}, 0}}\right)^{2+d_{\mathrm{p}} / d_{\mathrm{f}}}
$$

and

$$
D_{3 \mathrm{~d}} \approx D_{\mathrm{e}}\left(\frac{N}{N_{\mathrm{e}, 0}}\right)^{-2+\left(2-d_{\mathrm{p}}\right) / d_{\mathrm{f}}}
$$


which are generally applicable to the DFLA model, Grosberg's model, and the naive FLG model without tube dilation. The scaling exponents for the $N$ dependences of $\tau_{\text {relax }}$ and $D_{3 \mathrm{~d}}$ depend on the specific values of $d_{\mathrm{p}}$ and $d_{\mathrm{f}}$ in different models, as shown in Table 1.

2.2.3. Stress Relaxation and Viscosity-The stress relaxation modulus during entangled dynamics of nonconcatenated rings is

$$
G(t) \approx G_{\mathrm{e}}\left(\frac{t}{\tau_{\mathrm{e}}}\right)^{-\left[d_{\mathrm{f}}-(1-\theta) d_{\mathrm{P}}\right] /\left[2 d_{\mathrm{f}}+(1-\theta) d_{\mathrm{p}}+\theta\right]}, \text { for } \tau_{\mathrm{e}}<t<\tau_{\text {relax }}
$$

as derived in the Appendix (see eqs A.18 and A.19). If there is complete tube dilation with $\theta$ $=1$, the stress relaxation modulus is

$$
G(t) \approx G_{\mathrm{e}}\left(\frac{t}{\tau_{\mathrm{e}}}\right)^{-d_{\mathrm{f}} /\left(2 d_{\mathrm{f}}+1\right)}
$$

which is on the order of $k T$ per volume of $g(t) \approx N_{\mathrm{e}, 0}\left(t / \tau_{\mathrm{e}}\right)^{d_{\mathrm{f}} /\left(2 d_{\mathrm{f}}+1\right)}$ monomers (eq A.15 with $\theta=1)$. This result of $G(t)$ for complete tube dilation is independent of the fractal dimension $d_{\mathrm{p}}$ of the primitive path, reflecting the irrelevance of the fractal structure of the primitive path in the case of complete tube dilation. With $\theta=1$ and $d_{\mathrm{f}}=3$, the self-consistent FLG model predicts

$$
G(t) \approx G_{\mathrm{e}}\left(\frac{t}{\tau_{\mathrm{e}}}\right)^{-3 / 7}, \text { for } \tau_{\mathrm{e}}<t<\tau_{\text {relax }}
$$

If there is no tube dilation, we obtain from eq 44 that the stress relaxation modulus is

$$
G(t) \approx G_{\mathrm{e}}\left(\frac{t}{\tau_{\mathrm{e}}}\right)^{-\left(d_{\mathrm{f}}-d_{\mathrm{p}}\right) /\left(2 d_{\mathrm{f}}+d_{\mathrm{p}}\right)}, \text { for } \theta=0 \text { and } \tau_{\mathrm{e}}<t<\tau_{\text {relax }}
$$

which is general result for single-ring stress relaxation modulus applicable to the DFLA model, Grosberg's model, and the naive FLG model.

The derivations of the multi-ring stress relaxation moduli in the multi-ring DFLA model (see the extension of DFLA model to melts of multiple rings in section 1.3.1), Grosberg's model (see section 1.3.3), and the naive FLG model are not self-consistent. In these models, the multi-ring stress relaxation moduli correspond to $k T$ per volume of a characteristic loop containing $g(t) \approx N_{\mathrm{e}, 0}\left(t / \tau_{\mathrm{e}}\right)^{d_{\mathrm{f}} /\left(2 d_{\mathrm{f}}+d_{\mathrm{p}}\right)}$ monomers (eq A.15 with $\theta=0$ )

$$
G(t) \approx \frac{k T}{v g(t)} \approx G_{\mathrm{e}}\left(\frac{t}{\tau_{\mathrm{e}}}\right)^{-d_{\mathrm{f}} /\left(2 d_{\mathrm{f}}+d_{\mathrm{P}}\right)}, \text { for } \tau_{\mathrm{e}}<t<\tau_{\text {relax }}
$$

The derivation based on eq 48 is not self-consistent since it assumes complete tube dilation while uses the result of $g(t)$ obtained for the case of no tube dilation. A tube dilation exponent $\gamma=d_{\mathrm{f}} /\left(d_{\mathrm{f}}-d_{\mathrm{p}}\right)$ can be imposed to link the single-ring stress relaxation exponent $a_{\mathrm{s}}$ $=\left(d_{\mathrm{f}}-d_{\mathrm{p}}\right) /\left(2 d_{\mathrm{f}}+d_{\mathrm{p}}\right)$ in eq 47 and the multi-ring stress relaxation exponent $a=d_{\mathrm{f}} /\left(2 d_{\mathrm{f}}+d_{\mathrm{p}}\right)$ in eq 48 such that $a=\gamma a_{\mathrm{s}}$. The tube dilation exponent $\gamma$ depends on the values of $d_{\mathrm{f}}$ and $d_{\mathrm{p}}$. 
For the DFLA model with $d_{\mathrm{f}}=4$ and $d_{\mathrm{p}}=2$, the tube dilation exponent $\gamma=2$ is consistent with the approach of double reptation (eq 23), which has been applied to extend the original DFLA model to melts of nonconcatenated rings. ${ }^{38}$ For Grosberg's model with $d_{\mathrm{f}}=3$ and $d_{\mathrm{p}}$ $=5 / 3$ and the naive FLG model with $d_{\mathrm{f}}=3$ and $d_{\mathrm{p}}=2$, the corresponding tube dilation exponents $\gamma=9 / 4$ and $\gamma=3$ differ from $\gamma=2$ used for double reptation.

The results of the stress relaxation modulus $G(t)$ obtained from molecular dynamics simulations $^{47,48}$ are shown in Figure 6a. We simultaneously fit the simulation data of $G(t)$ for different $N$ to a crossover function

$$
G(t)=c_{1}\left(t^{-0.75}+c_{2} t^{-\alpha}\right) \exp \left[-t /\left(c_{3} N^{1 / \alpha}\right)\right]
$$

with the fitting parameters $c_{1}, c_{2}, c_{3}$, and $\alpha$ independent of $N$ (see Figure 6). This fitting procedure provides an estimate of the power-law stress relaxation in the asymptotic limit of large $N$ and hence allows evaluation of the predictions of various scaling models. Discussion of the finite- $N$ effects and comparison of different fitting procedures are presented in section B of the Supporting Information. The best fit of the simulation results of $G(t)$ yields that the scaling exponent for the power stress relaxation $a \approx 0.42 \pm 0.01$ (see eq A2 in the Supporting Information for the best fit values of $c_{1}, c_{2}$, and $c_{3}$ ). Accordingly, the relaxation time of a ring polymer increases with the degree of polymerization as $\tau_{\text {relax }} \sim N^{1 / a} \sim$ $N^{2.40 \pm 0.06}$. These results are in excellent agreement with the predictions of the selfconsistent FLG model that the exponent for power-law stress relaxation is $a=3 / 7 \approx 0.429$ and the exponent for relaxation time is $1 / a=7 / 3 \approx 2.33$ (see Table 1 ). All other models are in worse agreement with simulations as shown in Table 1.

The main rheological difference between melts of entangled linear chains and entangled rings is the absence of the plateau in the stress relaxation modulus $G(t)$ of the latter even for very high molar mass rings. Rubbery plateau is the most prominent feature of topological interactions in polymer liquids. ${ }^{1,5}$ The value of the plateau modulus $G_{\mathrm{e}} \approx k T /\left(v N_{\mathrm{e}, 0}\right)$ provides the operational definition and the most direct way of measuring the average degree of polymerization $N_{\mathrm{e}, 0}$ between entanglements. In contrast with entangled liquids (solutions and melts) of linear polymers, nonconcatenated ring polymers do not have rubbery plateau and are instead characterized by a power law stress relaxation function representing their self-similar dynamics.

Viscosity of melts is proportional to the product of relaxation time $\tau_{\text {relax }}$ and the terminal modulus $G\left(\tau_{\text {relax }}\right)$ at this time

$$
\eta \sim \tau_{\text {relax }} G\left(\tau_{\text {relax }}\right)
$$

For linear melts the product of reptation time (eq 36 for $\tau_{\text {relax }}$ can be used for linear polymers with $\theta=0, d_{\mathrm{f}}=2$, and $\left.d_{\mathrm{p}}=2\right)$ and plateau modulus $G_{\mathrm{e}} \approx k T /\left(v N_{\mathrm{e}, 0}\right)$ gives viscosity (see also eq 9 )

$$
\eta_{\text {linear }} \approx \eta_{\mathrm{e}}\left(\frac{N}{N_{\mathrm{e}, 0}}\right)^{3}
$$


where the viscosity at the crossover between entangled and unentangled melts with degree of polymerization $N_{\mathrm{e}, 0}$ is ${ }^{1}$

$$
\eta_{\mathrm{e}} \approx G_{\mathrm{e}} \tau_{\mathrm{e}} \approx \frac{\zeta b^{2} N_{\mathrm{e}, 0}}{v}
$$

The viscosity of nonconcatenated rings is

$$
\eta_{\text {ring }} \approx \eta_{\mathrm{e}}\left(\frac{N}{N_{\mathrm{e}, 0}}\right)^{1+2(1-\theta) d_{\mathrm{p}} / d_{\mathrm{f}}+\theta / d_{\mathrm{f}}}
$$

as derived in the Appendix (see eq A.24). The melt viscosity in the case of complete tube dilation

$$
\eta_{\text {ring }} \approx \eta_{\mathrm{e}}\left(\frac{N}{N_{\mathrm{e}, 0}}\right)^{1+1 / d_{\mathrm{f}}}, \text { for } \theta=1
$$

is independent of the fractal dimension $d_{\mathrm{p}}$ as the fractal structure of the primitive path is not felt by the ring with complete dilation. The self-consistent FLG model with $\theta=1$ and $d_{\mathrm{f}}=3$ predicts

$$
\eta_{\text {ring }} \approx \eta_{\mathrm{e}}\left(\frac{N}{N_{\mathrm{e}, 0}}\right)^{4 / 3}
$$

which is in excellent agreement with results of molecular dynamics simulations ${ }^{47,48}$ that give $\eta \sim N^{1.33 \pm 0.04}$ as shown in Figure 6b. With no tube dilation, eq 53 gives

$$
\eta_{\text {ring }} \approx \eta_{\mathrm{e}}\left(\frac{N}{N_{\mathrm{e}, 0}}\right)^{1+2 d_{\mathrm{p}} / d_{\mathrm{f}}}, \text { for } \theta=0
$$

The $N$ dependences of ring melt viscosity in the multi-ring DFLA model, Grosberg's model, and the naive FLG model are obtained in a manner that is not self-consistent. In each of these models, the terminal modulus is assumed to be on the order of $k T$ per ring $G\left(\tau_{\text {relax }}\right) \approx$ $k T /(v N) \approx G_{\mathrm{e}}\left(N / N_{\mathrm{e}, 0}\right)^{-1}$, and the viscosity of a ring melt is predicted to be

$$
\eta_{\text {ring }} \approx \eta_{\mathrm{e}}\left(\frac{N}{N_{\mathrm{e}, 0}}\right)^{1+d_{\mathrm{p}} / d_{\mathrm{f}}}
$$

based on eqs 42 and 50. However, the terminal modulus consistent with the terminal relaxation time given by eq 42 for no tube dilation should be $G\left(\tau_{\text {relax }}\right) \approx G_{\mathrm{e}}\left(N / N_{\mathrm{e}, 0}\right)^{-\left(1-d_{\mathrm{P}} / d_{\mathrm{f}}\right)} \approx\left(N / N_{\mathrm{e}, 0}\right)^{d_{\mathrm{P}} / d_{\mathrm{f}}} k T /(v N)$ (eq A.21 with $\left.\theta=0\right)$, which corresponds to $\left(N / N_{\mathrm{e}, 0}\right)^{d_{\mathrm{P}} / d_{\mathrm{f}}}>1$ times $k T$ per ring. As a result, eq 57 exhibits a weaker $N$ dependence than eq 56, as the terminal modulus is assumed to be as low as $k T$ per ring. The predictions of different models using eq 57 do not agree well with simulation results of ring viscosity as shown in Table 1 . 
Compared with the multi-ring DFLA model, Grosberg's model, and the naive FLG model, the self-consistent FLG model yields predictions of relaxation time $\tau_{\text {relax }}$, 3-dimensional diffusion constant $D_{3 \mathrm{~d}}$, stress relaxation function $G(t)$, and melt viscosity $\eta$ that agree the best with the corresponding simulation results (see Table 1). We also derive the time dependence of the mean-square displacement of monomers $\left\langle\Delta r^{2}(\mathrm{t})\right\rangle$ in section $\mathrm{D}$ of the Supporting Information. The self-consistent FLG model predicts that $\left\langle\Delta t^{2}(\mathrm{t})\right\rangle \sim t^{2 / 7}$ during the self-similar dynamics of rings at time interval $\tau_{\mathrm{e}}<t<\tau_{\text {relax }}$ (eq D.5 in Supporting Information), which is also in good agreement with simulation results as shown in Table 1 and Figure D.1 of the Supporting Information.

\section{SUMMARY}

The conformation of a nonconcatenated entangled ring is described as a fractal loopy globule (FLG) with fractal dimension $d_{f}=3$, in which loops at different length scales overlap with each other in a self-similar manner with constant Kavassalis-Noolandi overlap parameter $O_{\mathrm{KN}}=10-20$. A scaling model of the self-similar dynamics of rings as FLGs confined in tubes undergoing dilation has been developed. In our model, the tube diameter increases during the relaxation of entangled rings in such a way that it is comparable to both the root-mean-square center-of-mass displacement and the root-mean-square end-to-end distance of a characteristic loop. This picture corresponds to complete tube dilation and has been confirmed by our analysis of the primitive paths of entangled rings in molecular dynamics simulations. We derive a scaling theory of the entangled dynamics of nonconcatenated ring polymers with the extent of tube dilation characterized by the parameter $\theta$. In the case of complete tube dilation with $\theta=1$, the fractal dimension $d_{p}$ of the primitive path is not a relevant parameter, as a characteristic loop with its size on the order of tube diameter does not feel the fractal structure of the primitive path at length scales larger than the tube diameter. We show that traditional extensions of dynamic models without tube dilation to multi-ring dynamics with complete tube dilation are not self-consistent due to the lack of a renormalization of the friction coefficeint. In the traditional approach, complete tube dilation is put in artificially "by hand" as an afterthought and alters neither the relaxation time $\tau_{\text {relax }}$ nor the self-diffusion coefficient $\mathrm{D}_{3 \mathrm{~d}}$ (see section 2.2.2) but affects the stress relaxation function $G(t)$ and the melt viscosity $\eta$ (see section 2.2.3). We propose a self-consistent FLG model with an appropriate renormalization of the friction coefficient during complete tube dilation. The predictions of the self-consistent FLG model with $d_{\mathrm{f}}=3$ and $\theta=1$ are in the best agreement with the results of molecular dynamics simulations (see Table 1 and Figure 6).

In experiments, ${ }^{38,57}$ the power-law stress relaxation of entangled ring polymers has been observed with the apparent values of the stress relaxation exponent scattered between 0.4 and 0.5 for melts of polystyrene and polyisoprene rings with $N / N_{\mathrm{e}, 0}=6-13$ entanglements per ring. To determine the stress relaxation exponent more precisely, one needs to use much longer ring polymers to reduce the effects of finite polymer size as discussed in section A1 of the Supporting Information. The molecular weight dependence of ring melt viscosity $\eta \sim$ $\mathrm{N}^{2.2 \pm 0.3}$ identified in recent experiments ${ }^{57}$ is stronger than both molecular dynamics simulation results ${ }^{48}$ and various scaling model predictions (see Table 1). This discrepancy is likely due to minute contaminants of linear polymers in a ring melt, which enhance the melt 
viscosity with respect to that of pure rings. More systematic purification and characterization of ring polymers are needed to investigate the long time "foot" in the stress relaxation function and obtain a more accurate estimate of the molecular weight dependence of pure ring melt viscosity.

\section{Supplementary Material}

Refer to Web version on PubMed Central for supplementary material.

\section{Acknowledgments}

We thank Gary S. Grest and Jonathan D. Halverson for providing the equilibrated simulation samples of nonconcatenated ring polymers and help with analysis of the simulation data, Michael Kapnistos, Rossana Pasquino, and Dimitris Vlassopoulos for sharing the experimental data for polystyrene and polyisoprene ring polymers, and Dimitris Vlassopoulos, Alexander Grosberg, Ralf Everaers, Scott T. Milner, and Thomas C. B. McLeish for stimulating discussions. We acknowledge financial support from the National Science Foundation under Grants DMR-1309892, DMR-1436201, and DMR-1121107, the National Institutes of Health under Grants P01-HL108808 and 1UH2HL123645, and the Cystic Fibrosis Foundation.

\section{APPENDIX}

\section{Derivation of Stress Relaxation Function $G(t)$ and Viscosity}

We describe the self-similar dynamics of ring polymers in entangled melts with a quantitative characterization of tube dilation. A characteristic loop at time $t$ consists of $g(t)$ monomers and has just diffused by a distance of its size

$$
r(t) \approx a_{0}\left[\frac{g(t)}{N_{\mathrm{e}, 0}}\right]^{1 / d_{\mathrm{f}}}
$$

An entanglement strand at time $t$ contains

$$
N_{\mathrm{e}}(t) \approx N_{\mathrm{e}, 0}\left[\frac{g(t)}{N_{\mathrm{e}, 0}}\right]^{\theta} \approx N_{\mathrm{e}, 0}^{1-\theta}[g(t)]^{\theta}
$$

monomers with $0 \leq \theta \leq 1$. The parameter $\theta$ quantifies the extent of tube dilation. There is no tube dilation with $N_{\mathrm{e}}(\mathrm{t}) \approx N_{\mathrm{e}, 0}$ in the limit $\theta=0$, while complete tube dilation with $N_{\mathrm{e}}(t) \approx$ $g(t)$ corresponds to the limit $\theta=1$. The average spacing between topological constraints contributing to the tube at time $t$ is equal to the size of the corresponding entanglement strand

$$
a(t) \approx a_{0}\left[\frac{N_{\mathrm{e}}(t)}{N_{\mathrm{e}, 0}}\right]^{1 / d_{f}} \approx a_{0}\left[\frac{g(t)}{N_{\mathrm{e}, 0}}\right]^{\theta / d_{\mathrm{f}}}
$$

which is $a(t) \approx a_{0}$ for no tube dilation with $\theta=0$ and $a(t) \approx r(t)$ for complete tube dilation with $\theta=1$.

The tube dilation between times $t_{1}$ and $t_{2}>t_{1}$ with $t_{2} / t_{1}$ on the order of unity is illustrated in Figure $7 \mathrm{a}, \mathrm{b}$. The characteristic loop size increases from $r\left(t_{1}\right)$ to $r\left(t_{2}\right)$. Meanwhile, the number 
of monomers in an entanglement strand increases from $N_{\mathrm{e}}\left(t_{1}\right)$ to $N_{\mathrm{e}}\left(t_{2}\right)$, and the average spacing between the topological constraints contributing to the tube increases from $a\left(t_{1}\right)$ to $a\left(t_{2}\right)$. Using eqs A.1-A.3, we obtain the following relations

$$
\begin{aligned}
& \frac{r\left(t_{2}\right)}{r\left(t_{1}\right)} \approx\left[\frac{g\left(t_{2}\right)}{g\left(t_{1}\right)}\right]^{1 / d_{\mathrm{f}}} \\
& \frac{N_{\mathrm{e}}\left(t_{2}\right)}{N_{\mathrm{e}}\left(t_{1}\right)} \approx\left[\frac{g\left(t_{2}\right)}{g\left(t_{1}\right)}\right]^{\theta} \\
& \frac{a\left(t_{2}\right)}{a\left(t_{1}\right)} \approx\left[\frac{g\left(t_{2}\right)}{g\left(t_{1}\right)}\right]^{\theta / d_{\mathrm{f}}}
\end{aligned}
$$

The dynamics of a loop is described as the curvilinear diffusion of its center of mass along the primitive path (see red dashed lines in Figure 7a,b). Similar to the dynamics in the DFLA model shown in Figure 2b, effective curvilinear diffusion along the primitive path arises from the motions of only a small fraction of entanglement strands (see blue strands in Figure $7 \mathrm{a}, \mathrm{b}$ ) of the loop. We replace the monomer number $g$ of the loop in Figure $2 \mathrm{~b}$ and the corresponding monomer number $N_{\mathrm{e}}$ per entanglement strand respectively by $g(t)$ and $N_{\mathrm{e}}(t)$ and then use eq 19 to obtain the time it takes a loop to move by its size (eq A.1) and thus rearrange

$$
t \approx \tau_{\mathrm{e}}(t)\left[\frac{g(t)}{N_{\mathrm{e}}(t)}\right]^{2+d_{\mathrm{p}} / d_{\mathrm{f}}}
$$

where $\tau_{\mathrm{e}}(t)$ is the relaxation time of an entanglement strand at time $t$ and $d_{\mathrm{p}}$ is the fractal dimension of the primitive path of the loop. For the tube dilation between times $t_{1}$ and $t_{2}$ in Figure 7

$$
\frac{t_{2}}{t_{1}} \approx \frac{\tau_{\mathrm{e}}\left(t_{2}\right)}{\tau_{\mathrm{e}}\left(t_{1}\right)}\left[\frac{g\left(t_{2}\right)}{g\left(t_{1}\right)}\right]^{(1-\theta)\left(2+d_{\mathrm{p}} / d_{\mathrm{f}}\right)}
$$

which is obtained based on eqs A.5 and A.7.

We determine the relation between the two entanglement times $\tau_{\mathrm{e}}\left(t_{1}\right)$ and $\tau_{\mathrm{e}}\left(t_{2}\right)$ by relating the dynamics of entanglement strands at $t_{1}$ and $t_{2}$. An entanglement strand at $t_{1}$, which is termed as a $t_{1}$-entanglement-strand, moves by its size $a\left(t_{1}\right)$ during time $\tau_{\mathrm{e}}\left(t_{1}\right)$. Such a motion of a $t_{1}$-entanglement-strand acts as an elementary step of the dynamics of an entanglement strand at $t_{2}$, which we term as a $t_{2}$-entanglement-strand. The dynamics of a $t_{2}$-entanglementstrand can be described as the center-of-mass motion along its primitive path, which is a straight segment of size $a\left(t_{2}\right)$. Effective center-of-mass motion of a $t_{2}$-entanglement-strand only arises from the elementary steps of $t_{1}$-entanglement-strands along the primitive path of the $t_{2}$-entanglement-strand. The average number of $t_{1}$-entangle-ment-strands contributing to the effective center-of-mass motion of a $t_{2}$-entanglement-strand is 


$$
n \approx \frac{a\left(t_{2}\right)}{a\left(t_{1}\right)} \quad \text { (A.9) }
$$

Each of the $n t_{1}$-entanglement-strands causes mean-square center-of-mass displacement of the $t_{2}$-entanglement-strand along its primitive path by $\left[a\left(t_{1}\right) N_{\mathrm{e}}\left(t_{1}\right) / N_{\mathrm{e}}\left(t_{2}\right)\right]^{2}$ in an elementary time interval $\tau_{\mathrm{e}}\left(t_{1}\right)$. As a result, the diffusion coefficient for the center-of-mass motion of a $t_{2}$-entanglement-strand along its primitive path is

$$
D_{\mathrm{e}}\left(t_{2}\right) \approx \frac{n\left[a\left(t_{1}\right) N_{\mathrm{e}}\left(t_{1}\right) / N_{\mathrm{e}}\left(t_{2}\right)\right]^{2}}{\tau_{\mathrm{e}}\left(t_{1}\right)}
$$

The entanglement time $\tau_{\mathrm{e}}\left(t_{2}\right)$, during which a $t_{2}$ entanglement strand moves by its size $a\left(t_{2}\right)$, is

$$
\tau_{\mathrm{e}}\left(t_{2}\right) \approx \frac{\left[a\left(t_{2}\right)\right]^{2}}{D_{\mathrm{e}}\left(t_{2}\right)} \approx \tau_{\mathrm{e}}\left(t_{1}\right)\left[\frac{a\left(t_{2}\right)}{a\left(t_{1}\right)}\right]\left[\frac{N_{\mathrm{e}}\left(t_{2}\right)}{N_{\mathrm{e}}\left(t_{1}\right)}\right]^{2} \approx \tau_{\mathrm{e}}\left(t_{1}\right)\left[\frac{N_{\mathrm{e}}\left(t_{2}\right)}{N_{\mathrm{e}}\left(t_{1}\right)}\right]^{2+1 / d_{\mathrm{f}}}
$$

where eqs A.3, A.9, and A.10 were used. Combining eqs A.5, A.8 and A.11, one obtains

$$
t_{2} \approx t_{1}\left[\frac{g\left(t_{2}\right)}{g\left(t_{1}\right)}\right]^{2+(1-\theta) d_{\mathrm{p}} / d_{\mathrm{f}}+\theta / d_{\mathrm{f}}}
$$

From the relation in eq A.12, the time for a loop with $g$ monomers to diffuse by its size is

$$
\tau(g) \approx \tau_{\mathrm{e}, 0}\left(\frac{g}{N_{\mathrm{e}, 0}}\right)^{2+(1-\theta) d_{\mathrm{p}} / d_{\mathrm{f}}+\theta / d_{\mathrm{f}}}
$$

and the time for the entire ring containing $N$ monomers to diffuse by its size is

$$
\tau_{\mathrm{d}} \approx \tau_{\mathrm{e}, 0}\left(\frac{N}{N_{\mathrm{e}, 0}}\right)^{2+(1-\theta) d_{\mathrm{p}} / d_{\mathrm{f}}+\theta / d_{\mathrm{f}}}
$$

At time $\tau_{\mathrm{e}, 0}<t<\tau_{\mathrm{d}}$, a characteristic loop that has just diffused by its size contains

$$
g(t) \approx N_{\mathrm{e}, 0}\left(\frac{t}{\tau_{\mathrm{e}, 0}}\right)^{\rho} \quad(\mathrm{A} .15)
$$

monomers with $\rho \approx d_{\mathrm{f}} /\left[2 d_{\mathrm{f}}+(1-\theta) d_{\mathrm{p}}+\theta\right]$ according to eq A.13. Based on eq A.5, the number of monomers per entanglement strand increases with time as

$$
N_{\mathrm{e}}(t) \approx N_{\mathrm{e}, 0}\left(\frac{t}{\tau_{\mathrm{e}, 0}}\right)^{\theta \rho}, \text { for } \tau_{\mathrm{e}, 0}<t<\tau_{\mathrm{d}}
$$

The scaling relations in eq A.15 and A.16 are schematically shown in Figure 7c. 
The number of tube segments along the primitive path of a characteristic loop containing $g(t)$ monomers is $\left[g(t) / N_{\mathrm{e}}(t)\right]^{d_{\mathrm{p}} / d_{\mathrm{f}}}$ similar to $l / a$ in eq 13 , and the stress relaxation modulus

$$
G(t) \approx \frac{k T}{v g(t)}\left[\frac{g(t)}{N_{e}(t)}\right]^{d_{\mathrm{p}} / d_{\mathrm{f}}}, \text { for } \tau_{\mathrm{e}, 0}<t<\tau_{\mathrm{d}}
$$

where each unrelaxed tube segment contributes $k T$ per volume $v g(t)$ of the $g$-loop. Using eqs A.15-A.17, one obtains the stress relaxation modulus

$$
G(t) \approx G_{e}\left(\frac{t}{\tau_{\mathrm{e}, 0}}\right)^{-\left[d_{\mathrm{f}}-(1-\theta) d_{\mathrm{p}}\right] /\left[2 d_{\mathrm{f}}+(1-\theta) d_{\mathrm{p}}+\theta\right]}, \text { for } \tau_{\mathrm{e}, 0}<t<\tau_{\mathrm{d}}
$$

where $G_{\mathrm{e}} \approx k T /\left(v N_{\mathrm{e}, 0}\right)$ is the entanglement plateau modulus. The terminal relaxation of the remaining tube segments is analogous to the terminal relaxation of entangled linear polymers after the entanglement stress plateau. The diffusion time $\tau_{\mathrm{d}}$ and the time it takes for the tube segments remained at $t \approx \tau_{\mathrm{d}}$ to relax are comparable to each other, as they both correspond to the diffusion by the contour length of the primitive path at $t \approx \tau_{\mathrm{d}}$. Therefore, the terminal relaxation time of a ring (see eq A.14) is

$$
\tau_{\text {relax }} \approx \tau_{\mathrm{d}} \approx \tau_{\mathrm{e}, 0}\left(\frac{N}{N_{\mathrm{e}, 0}}\right)^{2+(1-\theta) d_{\mathrm{p}} / d_{\mathrm{f}}+\theta / d_{\mathrm{f}}}
$$

At $t>\tau_{\text {relax }}$, the stress relaxes exponentially with

$$
G(t) \approx G\left(\tau_{\text {relax }}\right) \exp \left(-\frac{t}{\tau_{\text {relax }}}\right)
$$

where the terminal modulus

$$
G\left(\tau_{\text {relax }}\right) \approx G_{\mathrm{e}}\left(\frac{N}{N_{\mathrm{e}, 0}}\right)^{-\left[1-(1-\theta) d_{\mathrm{p}} / d_{\mathrm{f}}\right]}
$$

based on eqs A.18 and A.19. Combining eqs A.18 and A.20, the stress relaxation function applicable to the entire time range above the entanglement time $\tau_{\mathrm{e}, 0}$ is

$$
G(t) \approx G\left(\tau_{\text {relax }}\right)\left(1+\frac{\tau_{\text {relax }}}{t}\right)^{\frac{d_{\mathrm{f}}-(1-\theta) / d_{\mathrm{p}}}{2 d_{\mathrm{f}}+(1-\theta) / d_{\mathrm{p}}+\theta}} \exp \left(-\frac{t}{\tau_{\text {relax }}}\right), \text { for } t>\tau_{\mathrm{e}, 0}
$$

For the original DFLA model with $d_{\mathrm{f}}=4, d_{\mathrm{p}}=2$, and $\theta=0$, the single-ring terminal relaxation is schematically shown by the red line in Figure 3. Note that the number of remaining tube segments is $\left(N / N_{\mathrm{e}, 0}\right)^{1 / 2}$ at $\tau_{\mathrm{d}} \approx \tau_{\mathrm{e}, 0}\left(N / N_{\mathrm{e}}\right)^{5 / 2}$.

Viscosity of nonconcatenated rings is calculated as an integral of the stress relaxation function in eq A.22

$$
\eta \approx \int_{0}^{\infty} G(t) \mathrm{d} t \sim \tau_{\text {relax }} G\left(\tau_{\text {relax }}\right)
$$


Using eqs A.19 and A.21, we obtain the viscosity of rings

$$
\eta \approx \eta_{\mathrm{e}}\left(\frac{N}{N_{\mathrm{e}, 0}}\right)^{1+2(1-\theta) d_{\mathrm{p}} / d_{\mathrm{f}}+\theta / d_{\mathrm{f}}}
$$

where $\eta_{\mathrm{e}} \approx \tau_{\mathrm{e}, 0} G_{\mathrm{e}}$.

There is an appropriate renormalization of monomeric friction during the tube dilation in entangled ring dynamics. Based on eqs A.6, A.9, and A.10, the curvilinear diffusion coefficient for a $t_{2}$-entanglement-strand $D_{\mathrm{e}}\left(t_{2}\right)$ is related to the curvilinear diffusion coefficient for a $t_{1}$-entanglement-strand $D_{\mathrm{e}}\left(t_{1}\right) \approx\left[a\left(t_{1}\right)\right]^{2} / \tau_{\mathrm{e}}\left(t_{1}\right)$ as

$$
D_{\mathrm{e}}\left(t_{2}\right) \approx D_{\mathrm{e}}\left(t_{1}\right)\left[\frac{N_{\mathrm{e}}\left(t_{2}\right)}{N_{\mathrm{e}}\left(t_{1}\right)}\right]^{-2+1 / d_{\mathrm{f}}} \quad \text { (A.25) }
$$

Using the Einstein relation eq 3, one obtains the relation between the frictions $\zeta_{\mathrm{e}}\left(t_{1}\right)$ and $\zeta_{\mathrm{e}}\left(t_{2}\right)$ of a $t_{1}$-entanglement-strand and a $t_{2}$-entanglement-strand

$$
\zeta_{\mathrm{e}}\left(t_{2}\right) \approx \zeta_{\mathrm{e}}\left(t_{1}\right) \frac{D_{\mathrm{e}}\left(t_{1}\right)}{D_{\mathrm{e}}\left(t_{2}\right)} \approx \zeta_{\mathrm{e}}\left(t_{1}\right)\left[\frac{N_{\mathrm{e}}\left(t_{2}\right)}{N_{\mathrm{e}}\left(t_{1}\right)}\right]^{2-1 / d_{\mathrm{f}}}
$$

The monomeric frictions at times $t_{1}$ and $t_{2}$ are $\zeta_{0}\left(t_{1}\right) \approx \zeta_{\mathrm{e}}\left(t_{1}\right) / N_{\mathrm{e}}\left(t_{1}\right)$ and $\zeta_{0}\left(t_{2}\right) \approx \zeta_{\mathrm{e}}\left(t_{2}\right) /$ $N_{\mathrm{e}}\left(t_{2}\right)$, respectively. Thus, the monomeric friction is renormalized as

$$
\zeta_{0}\left(t_{2}\right) \approx \zeta_{0}\left(t_{1}\right)\left[\frac{N_{\mathrm{e}}\left(t_{2}\right)}{N_{\mathrm{e}}\left(t_{1}\right)}\right]^{1-1 / d_{\mathrm{f}}} \quad(\text { A.27) }
$$

Furthermore, using eqs A.16 and A.27, we obtain the time dependence of monomeric friction coefficient

$$
\zeta_{0}(t) \approx \zeta_{0}\left(\tau_{\mathrm{e}, 0}\right)\left(\frac{t}{\tau_{\mathrm{e}, 0}}\right)^{\theta \rho\left(1-1 / d_{\mathrm{f}}\right)}
$$

For partial and complete tube dilation with $\theta>0$, the renormalized monomeric friction increases as tube dilates because $\theta \rho\left(1-1 / d_{\mathrm{f}}\right)>0$. Moreover, the renormalized monomeric friction increases more rapidly for a larger extent of tube dilation with a larger $\theta$. In the case of no tube dilation with $\theta=0$, there is no renormalization of monomeric friction, and $\zeta_{0}(t) \approx$ $\zeta_{0}\left(\tau_{\mathrm{e}}, 0\right) \approx \zeta_{0}$, where $\zeta_{0}$ is the bare monomeric friction.

\section{References}

1. Rubinstein, M.; Colby, RH. Polymer Physics. OUP; Oxford: 2003.

2. Kavassalis TA, Noolandi J. Phys Rev Lett. 1987; 59:2674-2677. [PubMed: 10035619]

3. Kavassalis TA, Noolandi J. Macromolecules. 1988; 21:2869-2879.

4. Kavassalis TA, Noolandi J. Macromolecules. 1989; 22:2709-2720.

5. Doi, M.; Edwards, SF. The Theory of Polymer Dynamics. Clarendon Press; Oxford: 1986.

6. Einstein A. Ann Phys. 1905; 322:549-560. 
7. Edwards SF. Proc Phys Soc, London. 1967; 91:513-519.

8. de Gennes, PG. Scaling Concepts in Polymer Physics. Cornell University Press; Ithaca, NY: 1979.

9. de Gennes PG. J Chem Phys. 1971; 55:572-579.

10. Doi M, Edwards SF. J Chem Soc, Faraday Trans 2. 1978; 74:1789.

11. Doi M, Edwards SF. J Chem Soc, Faraday Trans 2. 1978; 74:1802.

12. Doi M, Edwards SF. J Chem Soc, Faraday Trans 2. 1978; 74:1818.

13. Doi M, Edwards SF. J Chem Soc, Faraday Trans 2. 1978; 74:560.

14. Ferry, JD. Viscoelastic Properties of Polymers. Wiley; New York: 1980.

15. Doi M. J Polym Sci, Polym Lett Ed. 1981; 19:265.

16. Doi M. J Polym Sci, Polym Phys Ed. 1983; 21:667-684.

17. Lodge TP. Phys Rev Lett. 1999; 83:3218.

18. de Gennes PG. J Phys (Paris). 1975; 36:1199-1203.

19. Klein J. Macromolecules. 1978; 11:852.

20. Marrucci G. J Polym Sci, Polym Phys Ed. 1985; 23:159-177.

21. Viovy JL, Rubinstein M, Colby RH. Macromolecules. 1991; 24:3587-3596.

22. McLeish TCB. Adv Phys. 2002; 51:1379-1527.

23. Colby RH, Rubinstein M. Macromolecules. 1990; 23:2753-2757.

24. Milner S. Macromolecules. 2005; 38:4929-4939.

25. McLeish TCB. Nat Mater. 2008; 7:933-935. [PubMed: 19029927]

26. Rubinstein M. Talk in Leiden conference on "Genome mechanics at the nuclear scale". 2012

27. Obukhov SP, Rubinstein M, Colby RH. Macromolecules. 1994; 27:3191-3198.

28. Cremer T, Cremer C. Nat Rev Genet. 2001; 2:292. [PubMed: 11283701]

29. Halverson JD, Smrek J, Kremer K, Grosberg AY. Rep Prog Phys. 2014; 77:022601. [PubMed: 24472896]

30. de Gennes PG. J Phys Lett. 1985; 46:639-642.

31. Grosberg AY, Nechaev SK, Shakhnovich EI. J Phys (Paris). 1988; 49:2095-2100.

32. Grosberg, AY.; Khokhlov, AR. Statistical Physics of Macromolecules. American Institute of Physics; Woodbury, NY: 1994.

33. Graessley, WW. Polymeric Liquids \& Networks: Structure and Properties. Garland Science; 2004.

34. Wittmer J, Beckrich P, Johner J, Semenov A, Obukhov S, Meyer H, Baschnagel J. EPL. 2007; 77:56003.

35. Rubinstein M. Phys Rev Lett. 1986; 57:3023. [PubMed: 10033934]

36. Nechaev SK, Semenov AN, Koleva MK. Phys A (Amsterdam, Neth). 1987; 140:506-520.

37. Obukhov SP, Rubinstein M, Duke T. Phys Rev Lett. 1994; 73:1263. [PubMed: 10057666]

38. Kapnistos M, Lang M, Vlassopoulos D, Pyckhout-Hintzen W, Richter D, Cho D, Chang T, Rubinstein M. Nat Mater. 2008; 7:997-1002. [PubMed: 18953345]

39. Milner ST, Newhall JD. Phys Rev Lett. 2010; 105:208302. [PubMed: 21231269]

40. Cates ME, Deutsch JM. J Phys (Paris). 1986; 47:2121-2128.

41. Sakaue T. Phys Rev Lett. 2011; 106:167802. [PubMed: 21599414]

42. Brereton MG, Vilgis TA. J Phys A: Math Gen. 1995; 28:1149-1176.

43. Grosberg AY. Soft Matter. 2014; 10:560. [PubMed: 24652534]

44. Gutin AM, Grosberg AY, Shakhnovich EI. Macromolecules. 1993; 26:1293-1295.

45. Smrek J, Grosberg AY. J Phys: Condens Matter. 2015; 27:064117. [PubMed: 25563563]

46. Obukhov S, Johner A, Baschnagel J, Meyer H, Wittmer JP. EPL. 2014; 105:48005.

47. Halverson JD, Lee W-B, Grest GS, Grosberg YA, Kremer K. J Chem Phys. 2011; 134:204904. [PubMed: 21639474]

48. Halverson JD, Lee W-B, Grest GS, Grosberg YA, Kremer K. J Chem Phys. 2011; 134:204905. [PubMed: 21639475]

49. Staufer, D.; Aharony, A. Introduction to Percolation Theory. Taylor \& Francis Inc; Philadelphia, PA: 1994. 
50. Rosa A, Everaers R. Phys Rev Lett. 2014; 112:118302. [PubMed: 24702424]

51. Rubinstein M, Helfand E. J Chem Phys. 1985; 82:2477-2483.

52. Everaers R, Sukumaran SK, Grest GS, Svaneborg C, Sivasubramanian A, Kremer K. Science. 2004; 303:823-826. [PubMed: 14764875]

53. Read DJ, Jagannathan K, Likhtman AE. Macromolecules. 2008; 41:6843-6853.

54. McLeish TCB. J Rheol. 2003; 47:177.

55. Read DJ, Jagannathan K, Sukumaran SK, Auhl D. J Rheol. 2012; 56:823.

56. Shivokhin ME, van Ruymbeke E, Bailly C, Kouloumasis D, Hadjichristidis N, Likhtman AE. Macromolecules. 2014; 47:2451-2463.

57. Pasquino R, et al. ACS Macro Lett. 2013; 2:874-878. [PubMed: 26229737] 


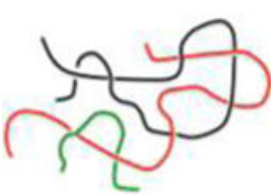

a

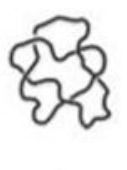

b

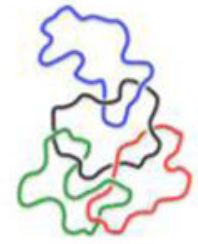

C

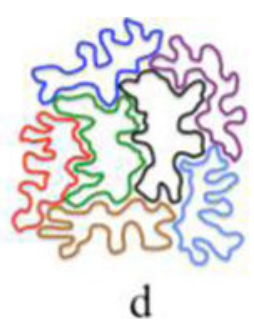

d

Figure 1.

(a) Temporary entanglements between linear chains. (b) Permanent self-knot of a ring. (c) Permanent links between rings. (d) Permanent topological constraints between nonconcatenated rings. 

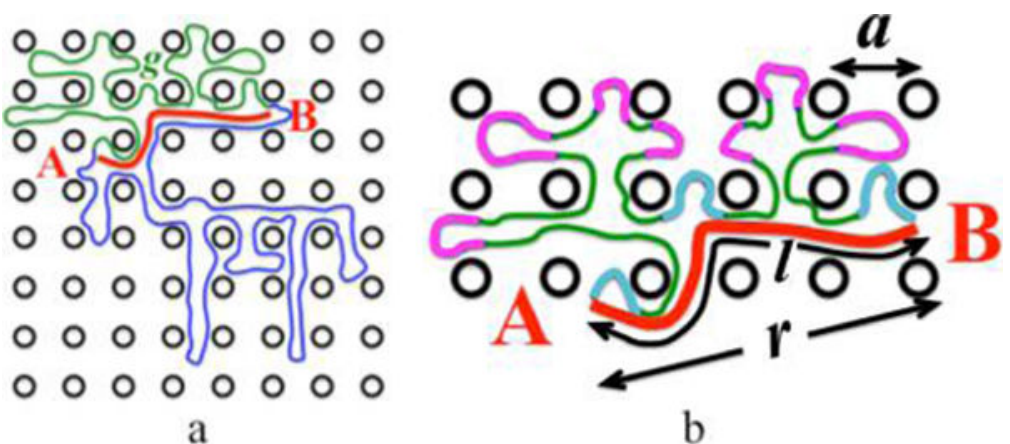

Figure 2.

(a) Double-folded lattice animal model of a non-concatenated ring in an array of fixed obstacles. Primitive path AB of ring section of $g$ monomers (green) is denoted by the red line. (b) Small fraction of elementary loops (cyan) move along the primitive path AB (red) leading to effective mass transport, while most of elementary loops (magenta) move along the double-folded sections (green), resulting in the change of conformations of these larger loops. The size of an elementary loop is on the order of the spacing a between fixed obstacles, while the size of the primitive path $\mathrm{AB}$ with contour length $l$ is $r \approx a(l / a)^{1 / 2} \approx$ $a\left(g / N_{\mathrm{e}}\right)^{1 / 4}$. 


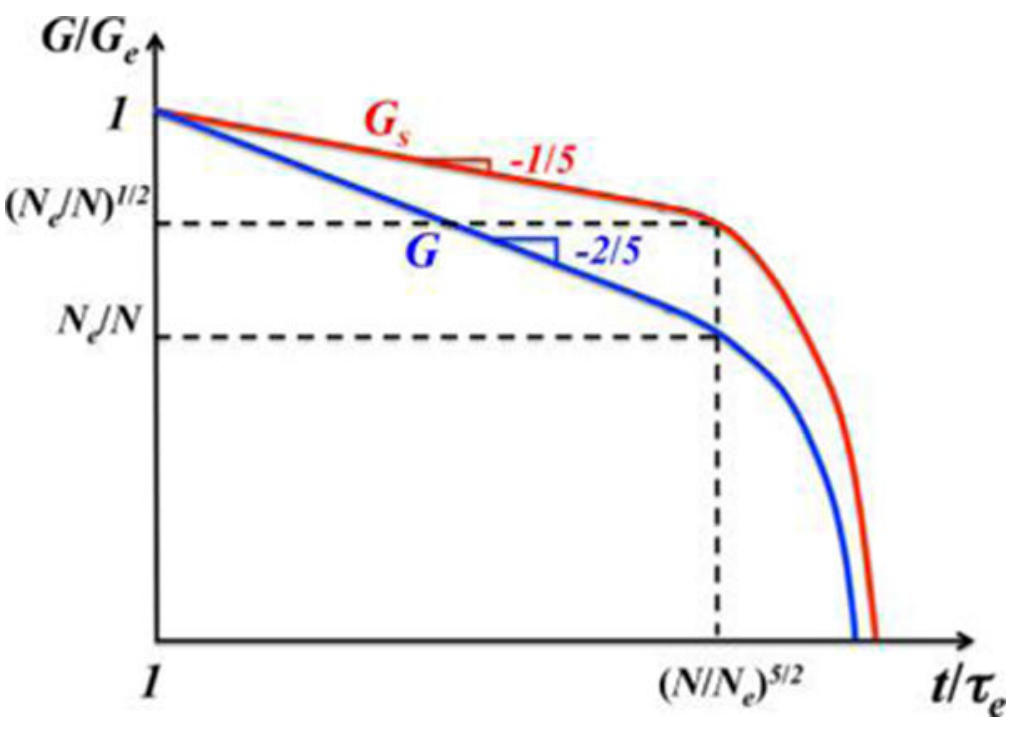

Figure 3.

Single-ring stress relaxation modulus $G_{\mathrm{S}}$ (red line) in the DFLA model and the corresponding multi-ring stress relaxation modulus $G$ (blue line) obtained by double reptation. Both $G_{\mathrm{s}}$ and $G$ are normalized by the entanglement plateau modulus $G_{\mathrm{e}}$ and are shown as functions of the time $t$ normalized by the entanglement time $\tau_{\mathrm{e}}$. Both axes are logarithmic. 


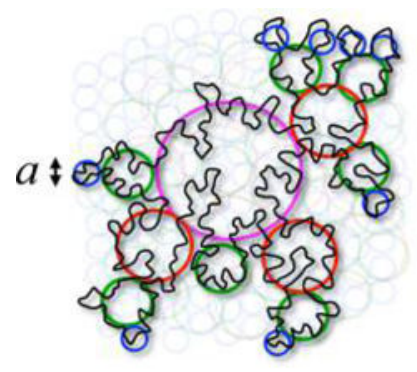

a

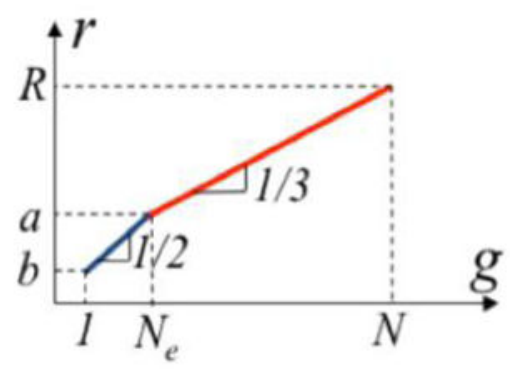

b

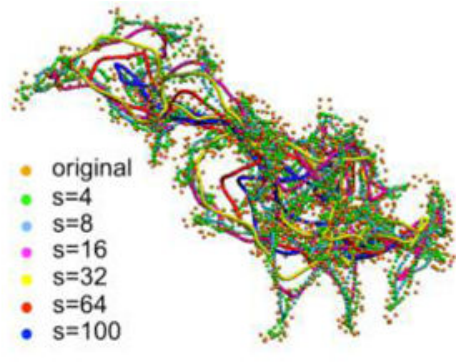

C

Figure 4.

(a) Schematic sketch of the fractal loopy globule (FLG) conformation of a ring (black line) in a melt of nonconcatenated rings. Regular circles of different colors indicate the length scales where loops of various sizes overlap with similar size neighbors at the same overlap parameter $O_{\mathrm{KN}}$. Circles associated with other rings are shown as the dimmed background. (b) Fractal structure in the FLG model: root-mean-square size $r$ of ring section with $g$ Kuhn segments on logarithmic scales. (c) Snapshot of a ring in a melt of nonconcatenated rings in molecular dynamics simulations 47,48 and the primitive paths obtained through pulling out loops consisting of less than $s$ monomers. 
a
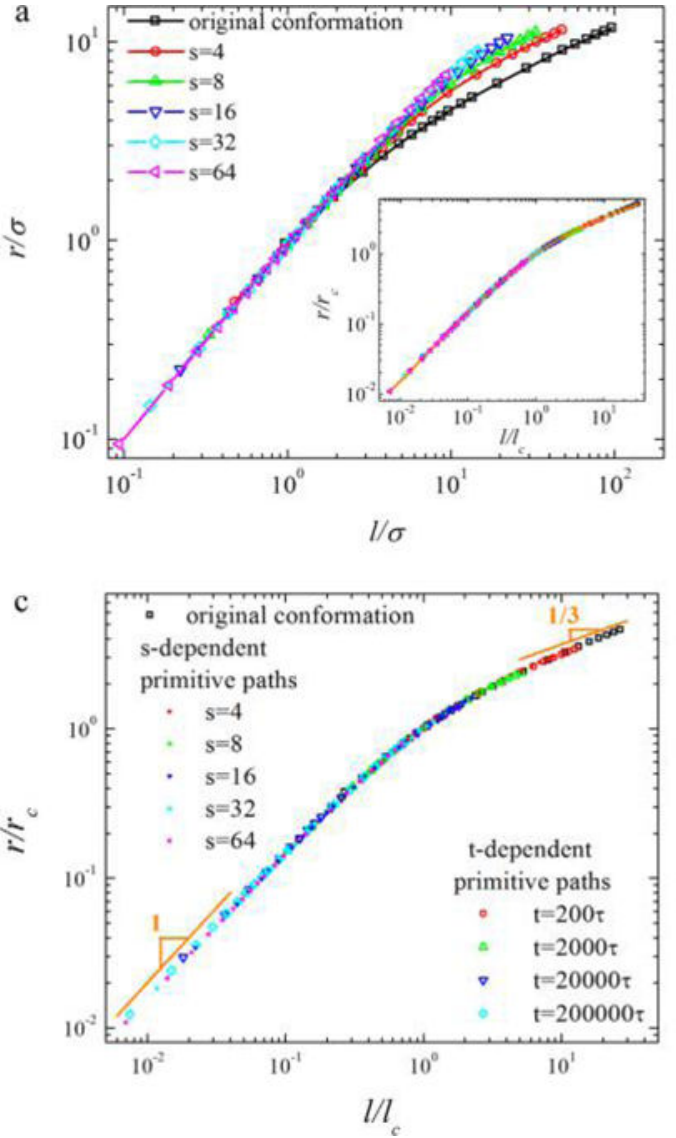

b

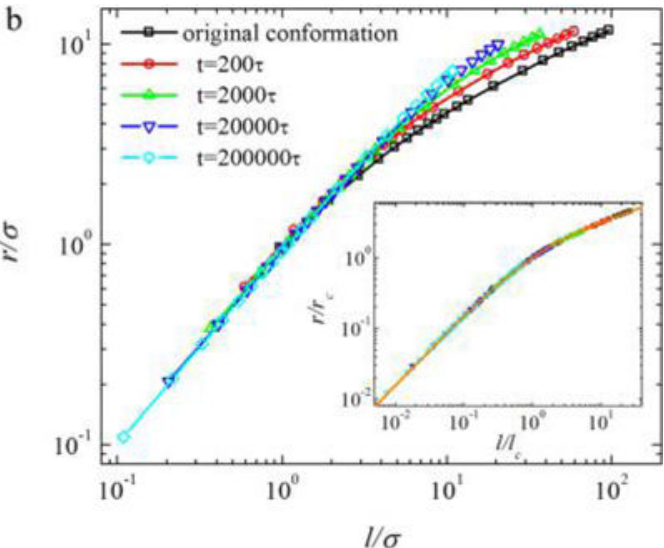

d

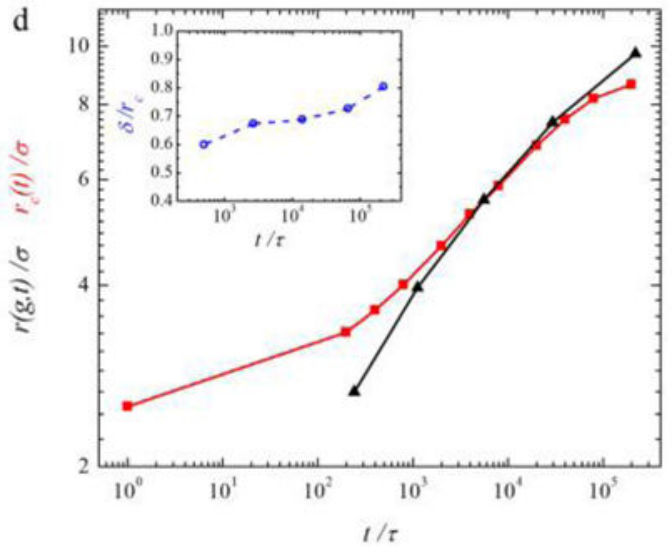

Figure 5.

Average size $r$ of a primitive path segment as a function of the contour length $l$ of the segment along (a) $s$-PP at indicated $s$ and (b) $t$-PP at indicated $t$. Results are obtained based on a molecular dynamics simulation ${ }^{47,48}$ of $M=200$ rings each with $N=1600$ beads. The insets show the primitive path segment contour length $l$ and size $r$ normalized by their respective crossover values $I_{\mathrm{c}}$ and $r_{\mathrm{c}}$. For both $s$-PP and $t$-PP, all normalized data points collapse onto corresponding master curves (orange lines). (c) Primitive path segment sizes $r$ normalized by the crossover values $r_{\mathrm{c}}$ as functions of the primitive path segment contour lengths $I$ normalized by the crossover values $l_{\mathrm{c}}$ for different $s$-PPs and $t$-PPs. (d) Crossover segment size $r_{\mathrm{c}}$ for $t$-dependent primitive paths (red squares) and the characteristic loop size $r(g, t)$ (black triangles) as functions of $t$. The inset shows the ratio $\delta / r_{\mathrm{c}}$ (blue circles) as a function of $t$, where $\delta$ is the root-mean-square deviation between pairs of $s$-PP and $t$-PP that have the same crossover segment size $r_{\mathrm{c}}$. 

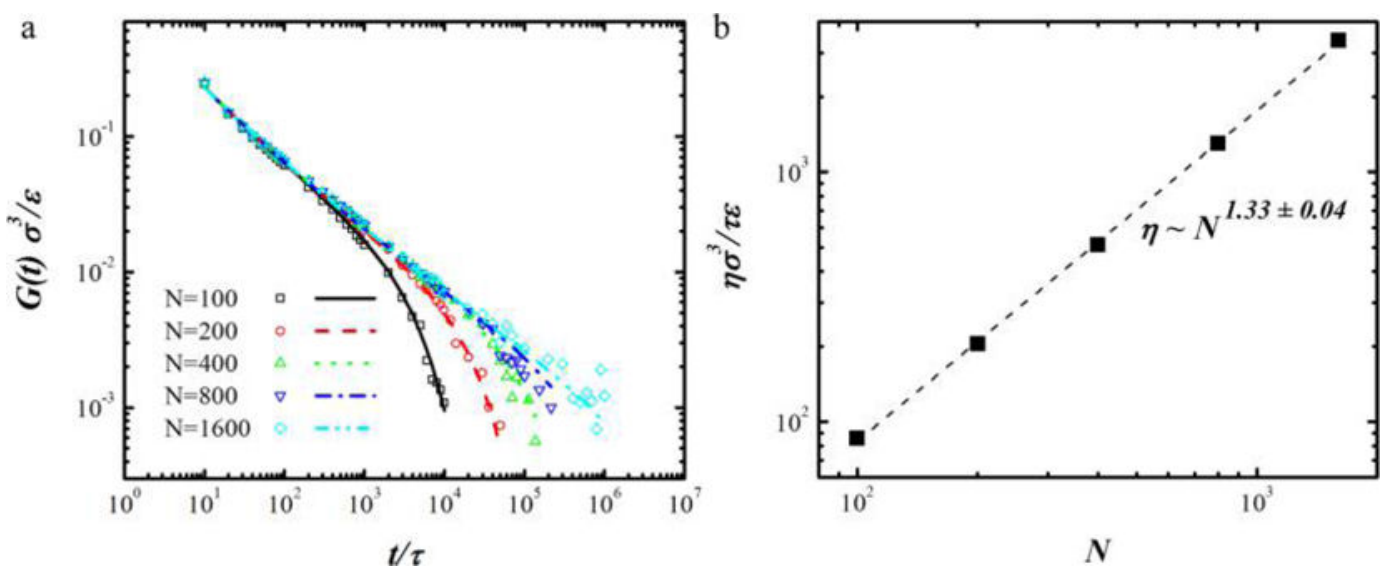

Figure 6.

(a) Stress relaxation modulus $G(t)$ in molecular dynamics simulations ${ }^{47,48}$ of nonconcatenated rings with different numbers of monomers per ring (empty symbols). The function in eq 49 is used to simultaneously fit all the simulation data for different $N$. The least-squares fit results are shown as lines. The best-fit value of the stress relaxation exponent describing the self-similar dynamics of rings in a melt is $a=0.42 \pm 0.01$, and accordingly the best-fit value of the relaxation time exponent is $1 / a=2.40 \pm 0.06$. (b) Viscosity $\eta$ of simulated rings scales with the degree of polymerization $N$ as $\eta \sim N^{1.33 \pm 0.04}$. 


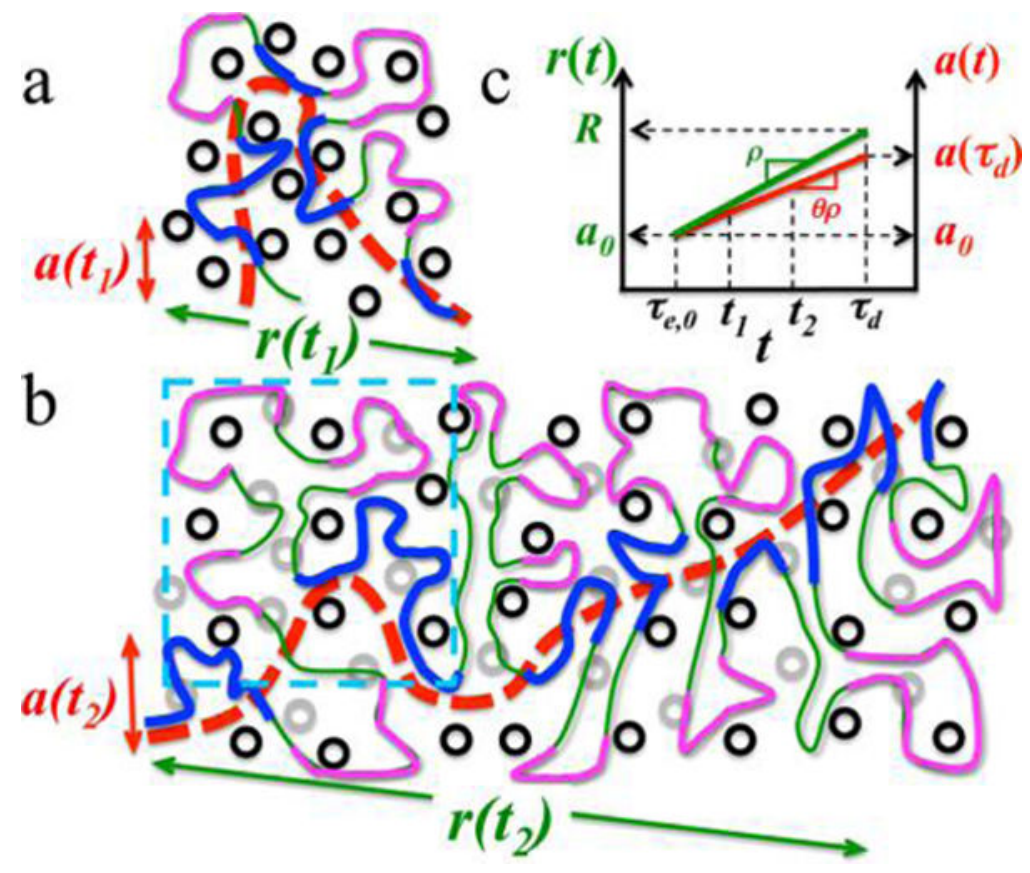

Figure 7.

(a) A characteristic loop of size $r\left(t_{1}\right)$ at time $t_{1}$ and (b) a characteristic loop of size $r\left(t_{2}\right)$ at time $t_{2}>t_{1}$ with the corresponding topological constraints (black circles) and primitive paths (dashed red lines). Chain section within the dashed-line cyan frame in (b) corresponds to the characteristic loop in (a). The topological constraints relevant to the confining tube at $t_{1}$ but irrelevant to the one at $t_{2}$ are shown as dimmed black circles in (b). The average spacing between topological constraints increases from $a\left(t_{1}\right)$ to $a\left(t_{2}\right)$. In both (a) and (b), only a small fraction of entanglement strands (blue) contribute to the effective diffusion along the primitive path (dashed red line), while most entanglement strands (magenta) do not make contributions. (c) Time dependences of the size $r(t)$ of a characteristic loop (green line) and the average spacing $a(t)$ between topological constraints (red line) for $\tau_{\mathrm{e}, 0}<t<\tau_{\mathrm{d}}$. Logarithmic scales. 


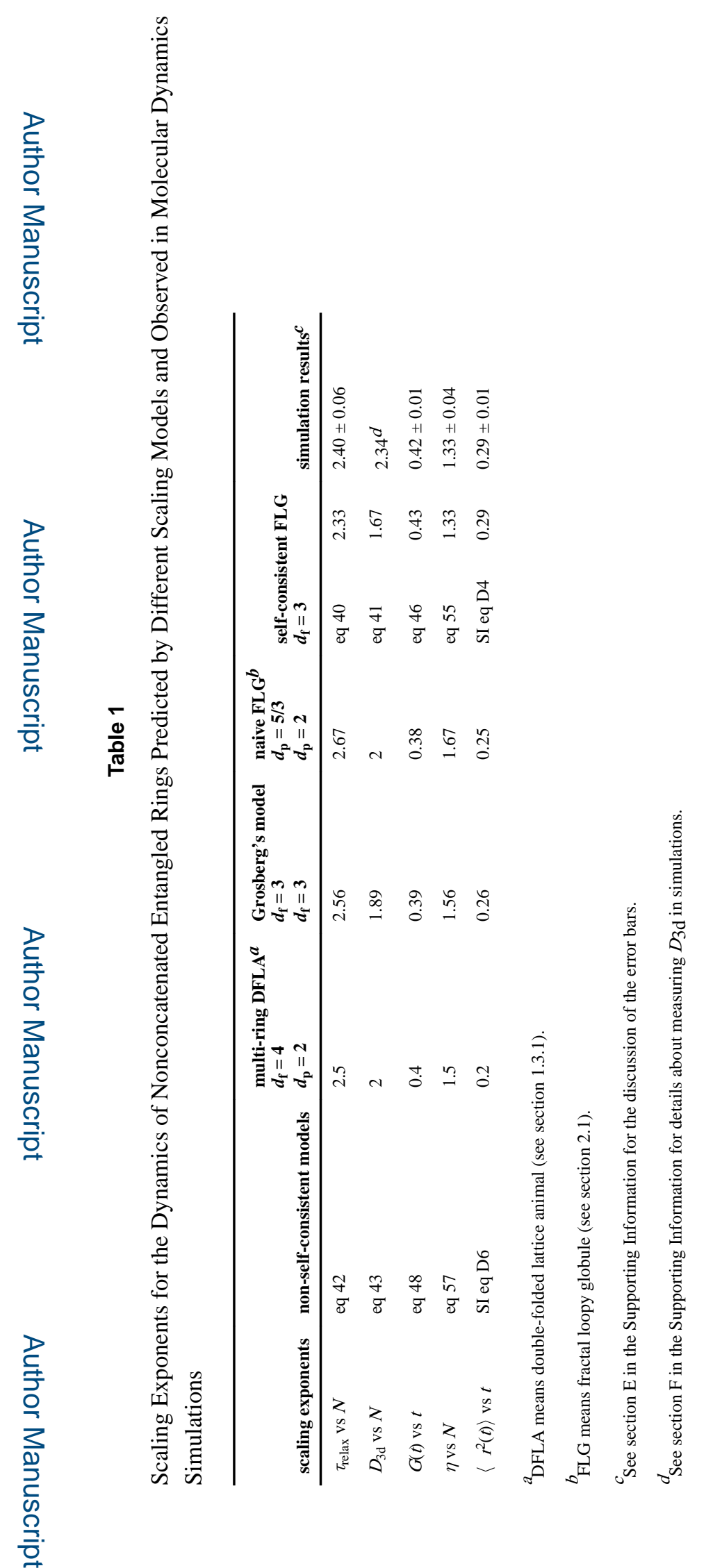

Macromolecules. Author manuscript; available in PMC 2017 January 01. 